\title{
The BUFFALO HST Survey
}

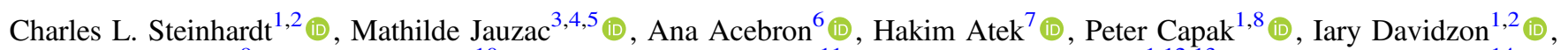
Dominique Eckert $^{9}$ (i), David Harvey ${ }^{10}$ (i), Anton M. Koekemoer ${ }^{11}$ (i), Claudia D. P. Lagos ${ }^{1,12,13}$ (1) , Guillaume Mahler ${ }^{14}$ (i),

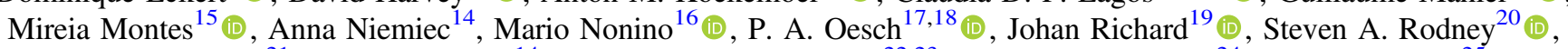
Matthieu Schaller $^{21}$ (1) , Keren Sharon ${ }^{14}$ (1) , Louis-Gregory Strolger ${ }^{22,23}$ (1) , Joseph Allingham $^{24}$ (1) , Adam Amara ${ }^{25}$ (1),

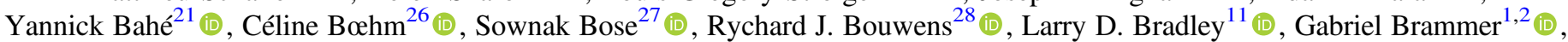
Tom Broadhurst ${ }^{29,30,83}$, Rodrigo Cañas ${ }^{13,31}$ (1) , Renyue $\mathrm{Cen}^{32}$ (10), Benjamin Clément ${ }^{33}$, Douglas Clowe ${ }^{34}$, Dan Coe $^{11}$ (1) ,

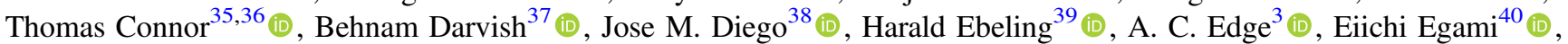
Stefano Ettori ${ }^{41,42}$ (10), Andreas L. Faisst ${ }^{8}$ (i) , Brenda Frye $^{43}$ (i) , Lukas J. Furtak $^{44}$ (D) , C. Gómez-Guijarro ${ }^{45}$ (i), J. D. Remolina González ${ }^{14}$ (1) , Anthony Gonzalez ${ }^{46}$ (ㅇ) , Or Graur $^{27,47,84}$ (i), Daniel Gruen ${ }^{48,49}$ (iD), David Harvey ${ }^{10}$, Hagan Hensley ${ }^{1,50}$, Beryl Hovis-Afflerbach ${ }^{1,51}$, Pascale Jablonka ${ }^{52,53}$, Saurabh W. Jha ${ }^{54}$ (1) , Eric Jullo ${ }^{55}$ (D) , Jean-Paul Kneib ${ }^{33,55}$ (D), Vasily Kokorev ${ }^{1,2}$ (10), David J. Lagattuta ${ }^{3,4,19}$ (10), Marceau Limousin ${ }^{56}$, Anja von der Linden ${ }^{57}$ (ㅇ), Nora B. Linzer ${ }^{1,51}$ (1), Adrian Lopez ${ }^{1,50}$, Georgios E. Magdis ${ }^{1,2,58}$ (1) , Richard Massey ${ }^{3}$ (10), Daniel C. Masters ${ }^{35}$ (1D), Matteo Maturi ${ }^{59}$, Curtis McCully ${ }^{60,61}$ (1D), Sean L. McGee ${ }^{62}$ (1) , Massimo Meneghetti ${ }^{63}$ (1), Bahram Mobasher ${ }^{64}$, Leonidas A. Moustakas ${ }^{35}$ (1), Eric J. Murphy ${ }^{65}$ (1), Priyamvada Natarajan ${ }^{66}$ (1), Mark Neyrinck ${ }^{67,68}$ (1) , Kyle O'Connor ${ }^{20}$, Masamune Oguri ${ }^{69,70,71}$ (1) , Amanda Pagul ${ }^{72}$ (1),

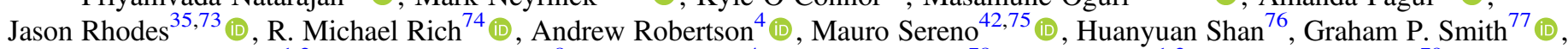
Albert Sneppen $^{1,2}$, Gordon K. Squires ${ }^{8}$, Sut-Ieng Tam ${ }^{4}$, Céline Tchernin ${ }^{78}$ (1) , Sune Toft ${ }^{1,2}$ (10), Keiichi Umetsu ${ }^{79}$ (1),

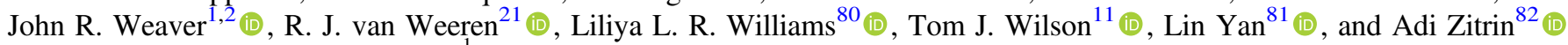

${ }^{1}$ Cosmic Dawn Center (DAWN), Denmark; steinhardt@nbi.ku.dk

${ }^{2}$ Niels Bohr Institute, University of Copenhagen, Lyngbyvej 2, Copenhagen Ø DK-2100, Denmark

${ }^{3}$ Centre for Extragalactic Astronomy, Durham University, South Road, Durham DH1 3LE, UK

${ }^{4}$ Institute for Computational Cosmology, Durham University, South Road, Durham DH1 3LE, UK

5 Astrophysics and Cosmology Research Unit, School of Mathematical Sciences, University of KwaZulu-Natal, Durban 4041, South Africa

${ }^{6}$ Physics Department, Ben-Gurion University of the Negev, P.O. Box 653, Be'er-Sheva 8410501, Israel

${ }^{7}$ Institut d'astrophysique de Paris, CNRS UMR7095, Sorbonne Université, 98 bis Boulevard Arago, F-75014 Paris, France

${ }^{8}$ IPAC, California Institute of Technology, 1200 E. California Blvd., Pasadena, CA 91125, USA

${ }^{9}$ University of Geneva, Ch. d'Ecogia 16, CH-1290 Versoix, Switzerland

${ }^{10}$ Lorentz Institute, Leiden University, Niels Bohrweg 2, Leiden, NL-2333 CA, The Netherlands

${ }^{11}$ Space Telescope Science Institute, 3700 San Martin Dr., Baltimore, MD 21218, USA

${ }^{12}$ International Centre for Radio Astronomy Research (ICRAR), University of Western Australia, 35 Stirling Hwy., Crawley, WA 6009, Australia

${ }^{13}$ ARC Centre of Excellence for All Sky Astrophysics in 3 Dimensions (ASTRO 3D), Australia

${ }^{14}$ Department of Astronomy, University of Michigan, 1085 S. University Ave., Ann Arbor, MI 48109, USA

${ }^{15}$ School of Physics, University of New South Wales, Sydney, NSW 2052, Australia

${ }^{16}$ INAF-Trieste Astronomical Observatory, Italy

${ }^{17}$ Department of Astronomy, University of Geneva, 51 Ch. des Maillettes, 1290 Versoix, Switzerland

${ }^{18}$ International Associate, Cosmic Dawn Center (DAWN), Switzerland

${ }^{19}$ Univ Lyon, Univ Lyon1, Ens de Lyon, CNRS, Centre de Recherche Astrophysique de Lyon UMR5574, F-69230, Saint-Genis-Laval, France

${ }^{20}$ University of South Carolina, Department of Physics \& Astronomy, Columbia, SC, USA

${ }^{21}$ Leiden Observatory, Leiden University, P.O. Box 9513, 2300 RA Leiden, The Netherlands

${ }^{22}$ Space Telescope Science Institute, USA

23 Johns Hopkins University, USA

${ }^{24}$ Sydney Institute for Astronomy, School of Physics, A28, The University of Sydney, NSW 2006, Australia

${ }^{25}$ Institute of Cosmology \& Gravitation, University of Portsmouth, Dennis Sciama Building, Portsmouth, PO1 3FX, UK ${ }^{26}$ University of Sydney, School of Physics, Sydney, NSW 2006, Australia

${ }^{27}$ Center for Astrophysics|Harvard \& Smithsonian, 60 Garden St., Cambridge, MA 02138, USA

${ }^{28}$ Leiden Observatory, Leiden University, NL-2300 RA Leiden, The Netherlands

${ }^{29}$ Department of Physics, University of the Basque Country, Spain

${ }^{30}$ Donostia International Physics Center, San Sebastian, Spain

${ }^{31}$ International Centre for Radio Astronomy Research, University of Western Australia, 35 Stirling Hwy., Crawley, WA 6009, Australia

${ }^{32}$ Department of Astrophysical Sciences, 4 Ivy Lane, Princeton, NJ 08544, USA

${ }^{33}$ Institute of Physics, Laboratory of Astrophysics, Ecole Polytechnique Fédérale de Lausanne (EPFL), Observatoire de Sauverny, 1290 Versoix, Switzerland

${ }^{34}$ Department of Physics \& Astronomy, Ohio University, Clippinger Labs 251B, Athens, OH 45701, USA

35 Jet Propulsion Laboratory, California Institute of Technology, Pasadena, CA 91109, USA

36 The Observatories of the Carnegie Institution for Science, 813 Santa Barbara St., Pasadena, CA 91101, USA

${ }^{37}$ Cahill Center for Astrophysics, California Institute of Technology, 1216 E. California Blvd., Pasadena, CA 91125, USA

${ }^{38}$ Instituto de Física de Cantabria (CSIC-UC). Edificio Juan Jordá. Avda Los Castros s/n. E-39005 Santander, Spain

${ }^{39}$ Institute for Astronomy, University of Hawaii, 2680 Woodlawn Dr., Honolulu, HI 96822, USA

${ }^{40}$ Steward Observatory, University of Arizona, 933 N. Cherry Ave., Tucson, AZ 85721, USA

${ }^{41}$ INAF, Osservatorio di Astrofisica e Scienza dello Spazio, via Pietro Gobetti 93/3, I-40129 Bologna, Italy

${ }^{42}$ INFN, Sezione di Bologna, viale Berti Pichat 6/2, I-40127 Bologna, Italy

${ }^{43}$ Department of Astronomy/Steward Observatory, University of Arizona, 933 N. Cherry Ave., Tucson, AZ 85721, USA

${ }^{44}$ Institut d'astrophysique de Paris, Sorbonne Université, CNRS UMR 7095, 98 bis bd Arago, F-75014 Paris, France

${ }^{45}$ Laboratoire AIM-Paris-Saclay, CEA/DSM-CNRS-Université Paris Diderot, Irfu/Service d'Astrophysique, CEA Saclay, Orme des Merisiers, F-91191 Gif-surYvette, France

${ }^{46}$ Department of Astronomy, University of Florida, 211 Bryant Space Center, Gainesville, FL 32611, USA

${ }^{47}$ Department of Astrophysics, American Museum of Natural History, New York, NY 10024, USA

${ }^{48}$ Kavli Institute for Particle Astrophysics and Cosmology, P.O. Box 2450, Stanford University, Stanford, CA 94305, USA 


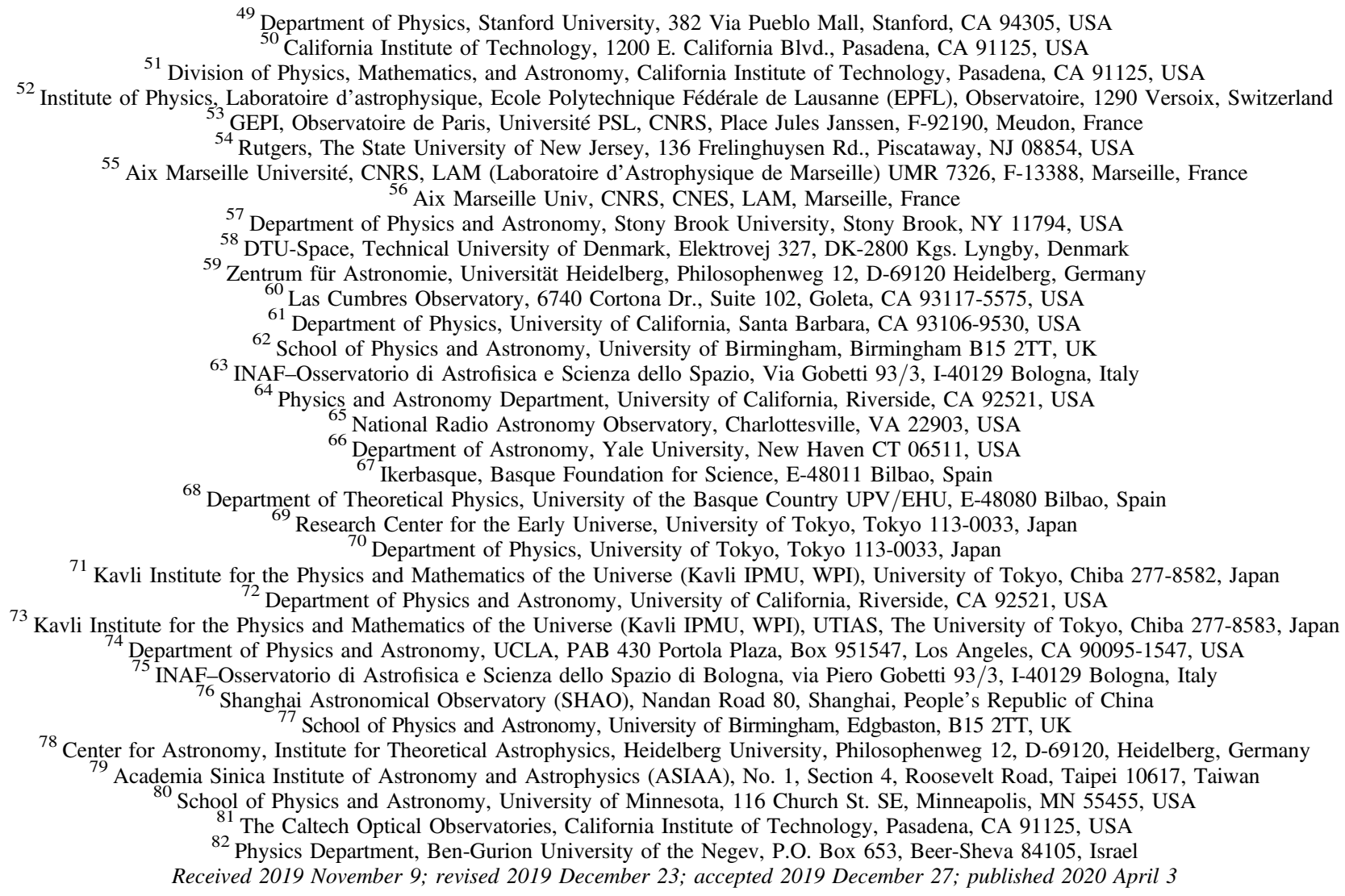

${ }^{49}$ Department of Physics, Stanford University, 382 Via Pueblo Mall, Stanford, CA 94305, USA

${ }^{50}$ California Institute of Technology, 1200 E. California Blvd., Pasadena, CA 91125, USA

${ }^{51}$ Division of Physics, Mathematics, and Astronomy, California Institute of Technology, Pasadena, CA 91125, USA

52 Institute of Physics, Laboratoire d'astrophysique, Ecole Polytechnique Fédérale de Lausanne (EPFL), Observatoire, 1290 Versoix, Switzerland

${ }_{53}$ GEPI, Observatoire de Paris, Université PSL, CNRS, Place Jules Janssen, F-92190, Meudon, France

${ }^{54}$ Rutgers, The State University of New Jersey, 136 Frelinghuysen Rd., Piscataway, NJ 08854, USA

55 Aix Marseille Université, CNRS, LAM (Laboratoire d'Astrophysique de Marseille) UMR 7326, F-13388, Marseille, France ${ }^{56}$ Aix Marseille Univ, CNRS, CNES, LAM, Marseille, France

${ }_{57}^{5}$ Department of Physics and Astronomy, Stony Brook University, Stony Brook, NY 11794, USA

${ }^{58}$ DTU-Space, Technical University of Denmark, Elektrovej 327, DK-2800 Kgs. Lyngby, Denmark

${ }^{59}$ Zentrum für Astronomie, Universität Heidelberg, Philosophenweg 12, D-69120 Heidelberg, Germany

${ }_{61}^{60}$ Las Cumbres Observatory, 6740 Cortona Dr., Suite 102, Goleta, CA 93117-5575, USA

${ }^{61}$ Department of Physics, University of California, Santa Barbara, CA 93106-9530, USA

${ }^{62}$ School of Physics and Astronomy, University of Birmingham, Birmingham B15 2TT, UK

${ }^{63}$ INAF-Osservatorio di Astrofisica e Scienza dello Spazio, Via Gobetti 93/3, I-40129 Bologna, Italy

${ }^{64}$ Physics and Astronomy Department, University of California, Riverside, CA 92521, USA

65 National Radio Astronomy Observatory, Charlottesville, VA 22903, USA

${ }^{66}$ Department of Astronomy, Yale University, New Haven CT 06511, USA

${ }^{67}$ Ikerbasque, Basque Foundation for Science, E-48011 Bilbao, Spain

${ }^{68}$ Department of Theoretical Physics, University of the Basque Country UPV/EHU, E-48080 Bilbao, Spain

${ }^{69}$ Research Center for the Early Universe, University of Tokyo, Tokyo 113-0033, Japan

${ }^{70}$ Department of Physics, University of Tokyo, Tokyo 113-0033, Japan

${ }^{71}$ Kavli Institute for the Physics and Mathematics of the Universe (Kavli IPMU, WPI), University of Tokyo, Chiba 277-8582, Japan

72 Department of Physics and Astronomy, University of California, Riverside, CA 92521, USA

${ }^{73}$ Kavli Institute for the Physics and Mathematics of the Universe (Kavli IPMU, WPI), UTIAS, The University of Tokyo, Chiba 277-8583, Japan

74 Department of Physics and Astronomy, UCLA, PAB 430 Portola Plaza, Box 951547, Los Angeles, CA 90095-1547, USA

5 INAF-Osservatorio di Astrofisica e Scienza dello Spazio di Bologna, via Piero Gobetti 93/3, I-40129 Bologna, Italy

${ }^{76}$ Shanghai Astronomical Observatory (SHAO), Nandan Road 80, Shanghai, People's Republic of China

${ }^{77}$ School of Physics and Astronomy, University of Birmingham, Edgbaston, B15 2TT, UK

${ }^{78}$ Center for Astronomy, Institute for Theoretical Astrophysics, Heidelberg University, Philosophenweg 12, D-69120, Heidelberg, Germany

${ }^{79}$ Academia Sinica Institute of Astronomy and Astrophysics (ASIAA), No. 1, Section 4, Roosevelt Road, Taipei 10617, Taiwan

${ }^{80}$ School of Physics and Astronomy, University of Minnesota, 116 Church St. SE, Minneapolis, MN 55455, USA

${ }_{82}$ The Caltech Optical Observatories, California Institute of Technology, Pasadena, CA 91125, USA

${ }^{82}$ Physics Department, Ben-Gurion University of the Negev, P.O. Box 653, Beer-Sheva 84105, Israel

Received 2019 November 9; revised 2019 December 23; accepted 2019 December 27; published 2020 April 3

\begin{abstract}
The Beyond Ultra-deep Frontier Fields and Legacy Observations (BUFFALO) is a 101 orbit +101 parallel Cycle 25 Hubble Space Telescope (HST) Treasury program taking data from 2018 to 2020. BUFFALO will expand existing coverage of the Hubble Frontier Fields (HFF) in Wide Field Camera 3/IR F105W, F125W, and F160W and Advanced Camera for Surveys/WFC F606W and F814W around each of the six HFF clusters and flanking fields. This additional area has not been observed by HST but is already covered by deep multiwavelength data sets, including Spitzer and Chandra. As with the original HFF program, BUFFALO is designed to take advantage of gravitational lensing from massive clusters to simultaneously find high-redshift galaxies that would otherwise lie below $H S T$ detection limits and model foreground clusters to study the properties of dark matter and galaxy assembly. The expanded area will provide the first opportunity to study both cosmic variance at high redshift and galaxy assembly in the outskirts of the large HFF clusters. Five additional orbits are reserved for transient followup. BUFFALO data including mosaics, value-added catalogs, and cluster mass distribution models will be released via MAST on a regular basis as the observations and analysis are completed for the six individual clusters.
\end{abstract}

Unified Astronomy Thesaurus concepts: Hubble Space Telescope (761); Galaxy clusters (584); Galaxy evolution (594); Gravitational lensing (670); Catalogs (205); Supernovae (1668); High-redshift galaxies (734)

\section{Introduction}

The Beyond Ultra-deep Frontier Fields and Legacy Observations (BUFFALO) program is a Hubble Space Telescope (HST) Treasury program expanding the Hubble Frontier Fields (HFF) galaxy cluster to adjacent areas that already benefit from ultradeep Spitzer and multiwavelength coverage. The program consists of 96 orbits +96 parallel orbits in the Frontier Fields and five orbits of planned supernova (SN) follow-up based upon expected rates.

The Frontier Fields program (Lotz et al. 2017) arose out of the realization that the same data set could be used to attack two of the most important questions in astronomy, even though

\footnotetext{
83 Ikerbasque Foundation.

${ }^{84}$ NSF Astronomy and Astrophysics Postdoctoral Fellow.
}

they were seemingly different topics. Gravitational lensing from massive, foreground clusters allows the $H S T$ to detect galaxies that would otherwise be too faint. At the same time, these observations allow us to model the dark matter distribution in the foreground clusters, which provides some of the leading constraints on the properties of dark matter.

BUFFALO will build upon the existing Frontier Fields programs by significantly broadening the area observed by HST around each of the six Frontier Fields clusters. BUFFALO will observe four times the area of the existing Frontier Fields in a tiling centered around the central region of each cluster field (and, in parallel, flanking field). BUFFALO will observe these new regions in five $H S T$ filters, including most of the filters used for the original Frontier Fields program. Observations over this broader region will provide significant improvements 
in our understanding of both dark matter and high-redshift galaxies.

Both of these studies benefit strongly from the presence of multiwavelength data that have been added to $H S T$ by the Spitzer Space Telescope (Werner et al. 2004), the Chandra $X$-ray Observatory (Weisskopf et al. 2000), the XMM-Newton $X$-ray Observatory (Jansen et al. 2001), and ground-based observatories. BUFFALO is designed to take advantage of this by expanding Frontier Fields coverage to fields in which these other data already exist.

The design benefits additionally from previous $H S T$ studies of high-redshift galaxies (McLure et al. 2010; Oesch et al. 2010; Bouwens et al. 2015, 2016; Finkelstein et al. 2015). Their measurements of the high-redshift luminosity function enable BUFFALO to choose a depth in five optical and nearinfrared passbands with the Advanced Camera for Surveys (ACS) and the Wide Field Camera 3 (WFC3) that is optimized to select the most luminous galaxies at $z>6$ and study their physical properties, such as stellar masses, by fully exploiting the preexisting, deep Spitzer/IRAC data around the Frontier Fields. BUFFALO is thus the logical extension of the HFF (Lotz et al. 2017), as well as previous HST+Spitzer extragalactic legacy surveys such as GOODS (Giavalisco et al. 2004), HUDF (Beckwith et al. 2006; Ellis et al. 2013; Illingworth et al. 2013; Labbé et al. 2015), CANDELS (Grogin et al. 2011; Koekemoer et al. 2011), S-CANDELS (Ashby et al. 2015), COSMOS (Scoville et al. 2007), SMUVS (Ashby et al. 2018), Brightest of Reionizing Galaxies (BoRG)/HIPPIES (Trenti et al. 2011; Yan et al. 2011), Cluster Lensing And Supernovae with Hubble (CLASH; Postman et al. 2012), SURFS UP (Bradač et al. 2014), or RELICS (Coe et al. 2019).

The BUFFALO observations and filter choices are summarized in Section 2. The BUFFALO collaboration includes groups that use a variety of techniques for mass modeling for individual clusters, as discussed in Section 3. In Section 4, the key science goals for high-redshift galaxy evolution are described. The improvements in our understanding of structure evolution and dark matter physics from expanding the $H S T$ footprint to a wider area around these clusters are discussed in Section 5. As discussed in Section 6, BUFFALO is also expected to observe a variety of transient objects, possibly including lensed SNe. Planned data products are described in Section 7. The survey program and key results are briefly summarized in Section 8.

The analysis presented here uses the $\mathrm{AB}$ magnitude system (Oke 1974; Gunn \& Stryker 1983) and a flat $\Lambda$ CDM cosmology with $\left(h, \Omega_{m}, \Omega_{\Lambda}\right)=(0.674,0.315,0.685)$ (Planck Collaboration et al. 2018) throughout.

\section{Observations}

The observing strategy for BUFFALO is designed to increase the area observed by $H S T$ around each of the six HFF clusters (Table 1) with both the ACS/WFC and WFC3/ IR cameras and in many of the same filter sets used for the HFF program (Figure 1). Specifically, each of the six HFF clusters is observed for a total of 16 orbits, divided into two orients (eight orbits each) differing by $180^{\circ}$ (approximately 6 months apart), thereby providing ACS/WFC and WFC3/IR coverage of both the main cluster field and the parallel field. In addition, each of the eight-orbit orients is divided into two epochs (at four orbits per epoch), separated by about 30 days, to enable searches for Type Ia $\mathrm{SNe}$ in both the main cluster and the parallel field.

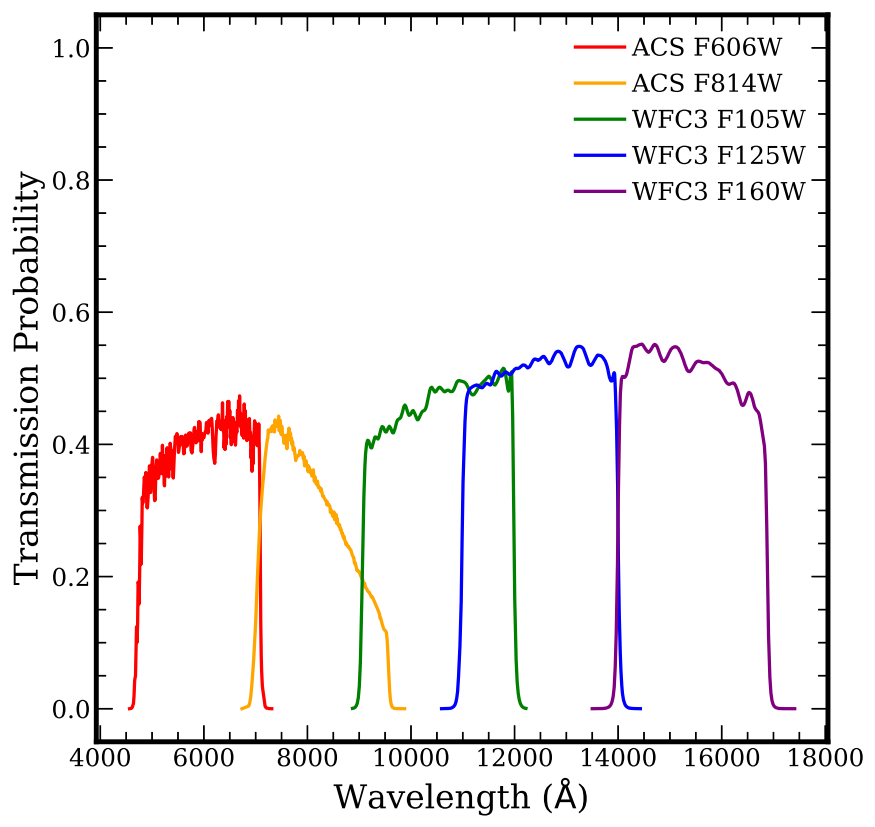

Figure 1. The HST filter profiles for BUFFALO observations using ACS and WFC3. BUFFALO uses many of the same filters as the original HFF program, with $2 / 3$ orbit depth in WFC3/F105W, WFC3/F125W, WFC3/F160W, and ACS/F606W and 4/3 orbit depth in ACS/F814W.

Table 1

BUFFALO/HFF Clusters and Flanking Fields

\begin{tabular}{lcc}
\hline \hline Field & Center (J2000) & $\begin{array}{c}\text { Flanking Field Center } \\
(\text { J2000) }\end{array}$ \\
\hline A370 & $02: 39: 52.9-01: 34: 36.5$ & $02: 40: 13.401: 37: 32.8$ \\
MACS & $07: 17: 34.0+37: 44: 49.0$ & $07: 17: 17.0+37: 49: 47.3$ \\
$\quad$ J0717.5+3745 & & \\
MACS & $04: 16: 08.9-24: 04: 28.7$ & $04: 16: 33.1-24: 06: 48.7$ \\
$\quad$ J0416.1-2403 & & \\
Abell S1063 & $22: 48: 44.4-44: 31: 48.5$ & $22: 49: 17.7-44: 32: 43.8$ \\
A2744 & $00: 14: 21.2-30: 23: 50.1$ & $00: 13: 53.6-30: 22: 54.3$ \\
MACS & $11: 49: 36.3+22: 23: 58.1$ & $11: 49: 40.5+22: 18: 02.3$ \\
$\quad$ J1149.5+2223 & & \\
\hline
\end{tabular}

Note. Please see Lotz et al. (2017) for additional information about the original HFF pointings and cluster properties.

Each four-orbit epoch consists of a $2 \times 2$ mosaic across the field, with spacing designed to maximize the area covered by the WFC3/IR detector, thereby covering four times the area of the original WFC3/IR imaging for both the main cluster and the parallel field in all cases. The corresponding ACS observations overlap each other by about half the field of view of the ACS, with the resulting mosaic covering three times the area of the original ACS imaging, again for both the main cluster and the parallel field (Figure 2).

Once all 16 orbits are obtained for each cluster, the resulting observations comprise a $2 \times 2$ mosaic of WFC $3 /$ IR data in F105W, F125W, and F160W, each at approximately $2 / 3$ orbit depth, utilizing a four-point dither to mitigate detector defects and provide subpixel sampling. The corresponding ACS/WFC observations consist of F606W at 2/3 orbit depth and F814W at $4 / 3$ orbit depth, again utilizing a four-point dither to mitigate detector defects, provide subpixel sampling, and step across the gap between the two chips in ACS. For each of the six clusters, 


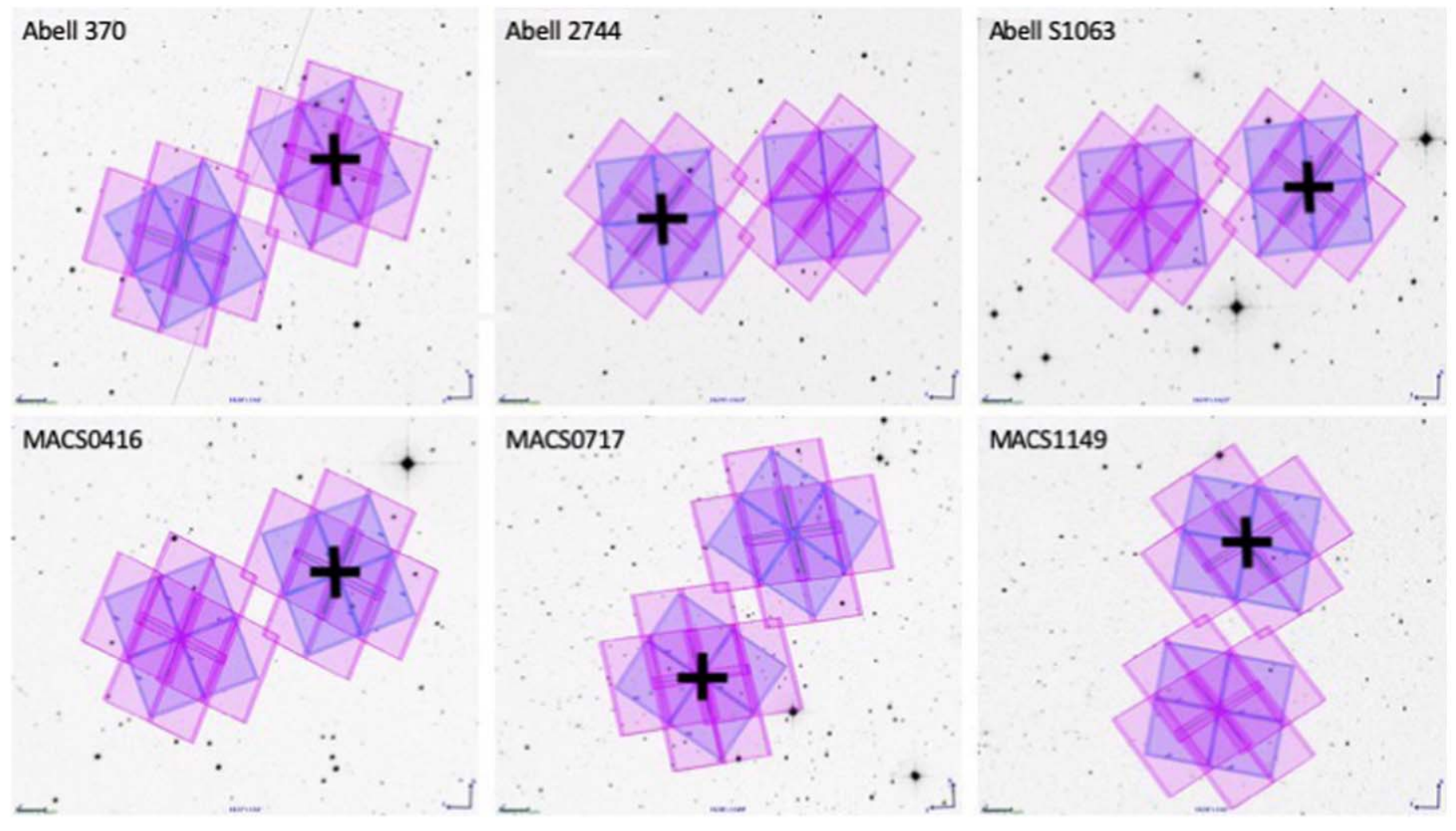

Figure 2. BUFFALO coverage map for the six Frontier Field clusters, produced by running the HST Astronomer's Proposal Tool on the full set of observations from this program (ID 15117). Each BUFFALO pointing, chosen to overlap the regions with deep channel 1 and 2 data from Spitzer/IRAC, is covered by $2 / 3$ orbit depth WFC3 exposures in F105W, F125W, and F160W (blue) and ACS observations taken in parallel at 2/3 orbit depth in F606W and 4/3 orbit depth in F814W (pink). The ACS imaging fully covers the WFC3-IR area and goes somewhat beyond it due to the larger field of view of the ACS. The new BUFFALO tiling is centered at the central region of each cluster field, indicated with a black plus sign, with parallel observations similarly expanding the existing flanking fields.

the final data set therefore consists of 160 exposures in total, at four exposures per pointing, over eight different pointings (four on the main cluster and four on the parallel field), for each of the five ACS/WFC3 filters used in the program.

As each new set of observations was obtained, it was downloaded and processed with a series of steps that go beyond the standard STScI pipeline processing. In particular, these additional steps included refining the astrometry for all exposures to accuracies better than milliarcsecond-level, as well as improved background-level estimates, refined cosmicray rejection, and improved treatment of time-variable sky and persistence removal for the WFC3/IR detectors. These improvements all followed similar procedures to those described in Koekemoer et al. (2011), where more details on these steps are presented. Exposures in the F105W band are affected by time-varying background signals due to variable atmospheric emission during the exposure. As a result, the background of an exposure is higher, and the final depth of the images can be impacted. For its characteristics, diffuse and extended, this effect can impact the detection of intracluster light (ICL; see Section 5.4) in this band. The BUFFALO image processing corrects for the time-variable background by either subtracting a constant background for each readout or excluding the affected frames (see Koekemoer et al. 2013 for more details on the processing). A preliminary inspection of the data shows no difference between the ICL maps for the F105W and the other IR bands.

The final products prepared from these procedures included full-depth combined mosaics for each filter, as well as the individual exposures necessary for lensing models, all astrometrically aligned and distortion-rectified onto a common astrometric pixel grid that was tied directly to the GAIA-DR2 absolute astrometric frame using catalogs provided by $\mathrm{M}$. Nonino (2020, private communication). The final mosaics produced from this process also included all other previous $H S T$ data obtained on these fields in these filters and therefore are the deepest $H S T$ mosaics available for these fields.

\subsection{Analysis Tools and Methods}

In order to allow analysis of the intrinsic properties of background lensed galaxies, the BUFFALO survey follows the HFF philosophy of releasing high-level data products through the Mikulski Archive for Space Telescopes (MAST) for the scientific community. The BUFFALO team will produce several strong, weak, and joint strong+weak lensing models from independent teams using different techniques described further in Section 3. BUFFALO will then deliver mass, magnification, deflection, and associated error maps for different redshifts provided by each independent modeling team of the collaboration, as well as weak lensing catalogs. An online magnification calculator will also be available for fast magnification estimates and errors within the modeled fields of view for each modeling pipeline. The BUFFALO data products are available at doi:10.17909/t9-w6tj-wp63.

\section{Lensing Mass Modeling}

The BUFFALO survey extends the HST coverage of the six HFF (Lotz et al. 2017) clusters by a factor of about 4 , which will expand the measurements of the overall mass profile and 
substructure characteristics of both the dark and luminous components of galaxy clusters up to $\sim 3 / 4 \times R_{\text {vir. }}$. Recent studies have shown that the shear induced by distant and massive substructures can extend to the cluster core (Acebron et al. 2017; Mahler et al. 2018; Lagattuta et al. 2019), and therefore including them in the overall mass modeling can affect the central component of the model and remove some potential biases. Mahler et al. (2018) showed that structures in the outskirts of A2744 found with a weak lensing analysis (Jauzac et al. 2016a) impact the mass measurements in the cluster core, which in turn affects the magnification estimates of high-redshift sources. Lagattuta et al. (2019) studied A370 by extending the Multi-Unit Spectroscopic Explorer mosaic around A370 together with deeper imaging data from the HFF program. Their best-fit strong lensing model needed a significantly large external shear term that is thought to come from structures along the line of sight and at larger projected radii in the lens plane. Moreover, Acebron et al. (2017) analyzed the impact of distant and massive substructures on the overall mass and density profile reconstruction with $H F F$-like simulated clusters (Meneghetti et al. 2017). They found that the density profile in the outskirts of the cluster $(\geqslant 600 \mathrm{kpc})$ was underestimated by up to $\sim 30 \%$. Including the substructures in the modeling helps to better constrain the mass distribution, with an improvement between $\sim 5 \%$ and $\sim 20 \%$ in the most distant regions of the cluster. Further, the bias introduced by these unmodeled structures also affected the retrieval of cosmological parameters with the strong lensing cosmography technique.

Mapping these structures is essential not only for improving the accuracy of the mass models but also to gain a better understanding of the physical properties governing galaxy clusters. Indeed, at the densest regions of the universe and the sites of constant growth due to their location at the nodes of the cosmic web, galaxy clusters represent a privileged laboratory for testing the properties of dark matter and improving our global picture of structure evolution. These recent studies have thus further proven the need for extended data coverage around galaxy clusters.

With its extended spatial coverage of the HFF clusters, BUFFALO will provide unprecedented weak lensing measurements thanks to HST high-resolution imagery, allowing for precise measurement of the shapes of weakly lensed galaxies. The combination of BUFFALO weak lensing and HFF strong lensing analyses will provide the most precise mass measurements ever obtained for those clusters. BUFFALO will detect structures that can only be significantly detected with $H S T$ weak lensing. While residing in the cluster outskirts, they still introduce a significant bias in the mass measurements, as explained earlier, and thus on the magnification estimates that are crucial for high-redshift studies.

Following the success of a similar program with the original HFF observations, the lensing profile in BUFFALO will be modeled by several independent teams, with each result released for public use (Section 4). The aim of the BUFFALO mass modeling challenge is to bring a better understanding of the behavior of these clusters, as well as the different systematic errors arising from the different techniques or assumptions used (Meneghetti et al. 2017). The algorithms participating in the modeling challenge are briefly described below.
1. The glafic (Oguri 2010) code performs mass modeling adopting a parametric approach. Halo components are remodeled by a Navarro-Frenk-White (NFW) profile, and cluster member galaxies are modeled by a pseudoJaffe profile. External perturbations are added for better modeling. A detailed description of the mass modeling of the $H F F$ clusters with glafic is given in Kawamata et al. (2016, 2018), indicating that positions of multiple images are reproduced with a typical accuracy of $\sim 0$ ". 4 .

2. Grale (Liesenborgs et al. 2006, 2009) is a flexible, freeform method based on a genetic algorithm that uses an adaptive grid to iteratively refine the mass model. As input, it uses only the information about the lensed images and nothing about the cluster's visible mass. This last feature sets Grale apart from most other lens mass reconstruction techniques and gives it the ability to test how well mass follows light on both large and small scales within galaxy clusters. Grale's description, software, and installation instructions are available online at http://research.edm.uhasselt.be/ jori/grale.

3. LENSTOOL (Jullo et al. 2007; Jullo \& Kneib 2009) utilizes a Bayesian Markov Chain Monte Carlo sampler to optimize the model parameters using the positions and spectroscopic redshifts (as well as magnitudes, shapes, and multiplicity, if specified) of the multiply imaged systems. The overall matter distribution of clusters is decomposed into smooth large-scale components and the small-scale halos associated with the locations of cluster galaxies.

A new version of LENSTOOL (A. Niemiec et al. 2020, in preparation) allows the combination of a parametric strong lensing modeling of the cluster's core, where the multiple images appear, with a more flexible, nonparametric weak lensing modeling at larger radii, the latter leading to the detection and characterization of substructures in the outskirts of galaxy clusters (Jauzac et al. 2016a, 2018).

4. The light-traces-mass (LTM; Broadhurst et al. 2005; Zitrin et al. 2009) methodology is based on the assumption that the underlying dark matter distribution in the cluster is traced by the distribution of the luminous component, i.e., the cluster galaxies and their luminosities. Only a small number of free parameters is needed to generate a mass model where the position and source redshift of multiple images are used as constraints. The LTM methodology has proven to be a powerful method for identifying new multiple images, as well as for constraining the cluster mass distribution (Merten et al. 2011; Zitrin et al. 2015; Frye et al. 2019). This pipeline also allows the incorporation of other constraints, including weak lensing shear measurements, time delays, or relative magnifications.

5. The WSLAP+ (Diego et al. 2007, 2016a) method falls in the category of hybrid methods. The mass distribution is decomposed into two components: (1) a grid component (free-form method) that accounts for the diffuse mass distribution and (2) a compact component (parametric method) that accounts for the mass associated with the member galaxies. The method combines strong lensing arc positions with weak lensing measurements when available. The solution is found by minimizing the quadratic form of a system of linear equations. The role 
of the regularization is adopted by the number of iterations in the minimization code, although, if resolved lensed systems are present (i.e., arcs with multiple identifiable knots), the solutions derived are very robust and weakly dependent on the number of iterations. Details can be found in Diego et al. (2007, 2016a) and references therein.

6. The CLUster lensing Mass Inversion (CLUMI) code (Umetsu 2013) combines wide-field weak lensing (shear and magnification) constraints for reconstructing binned surface mass density profiles, $\Sigma(R)$. This code has been used for the CLASH weak lensing studies (e.g., Umetsu et al. 2014). It can also include central strong lensing constraints in the form of the enclosed projected total mass, as done in Umetsu et al. (2016).

The CLUMI 2D code (Umetsu et al. 2018) is a generalization of CLUMI into a $2 \mathrm{D}$ description of the pixelized mass distribution. It combines the spatial 2D shear pattern (g1, g2) with azimuthally averaged magnification-bias measurements, which impose a set of azimuthally integrated constraints on the $\Sigma(x, y)$ field, thus effectively breaking the mass-sheet degeneracy. It is designed for an unbiased reconstruction of both mass morphology (e.g., halo ellipticity) and the radial mass profile of the projected cluster matter distribution.

\section{Impact on Studies of the High-redshift Universe}

Massive galaxies at $z \sim 8-10$ are in the midst of a critical transition between initial collapse and subsequent evolution (Steinhardt et al. 2014, 2016; Bouwens et al. 2015; Mashian et al. 2016). Galaxies are thought to form via hierarchical merging, building from primordial dark matter fluctuations into massive protogalaxies (Springel et al. 2005; Vogelsberger et al. 2014). Indeed, at $z<6$, the stellar mass function (SMF) is well approximated by a Schechter function with a clear exponential cutoff above a characteristic mass.

However, if early galactic assembly is dominated by simple baryonic cooling onto dark matter halos, at very high redshifts, the SMF should be a Press-Schechter-like power law with no exponential cutoff. Similarly, studies of star-forming galaxies at $z<6$ (Daddi et al. 2007; Noeske et al. 2007; Lee et al. 2013; Speagle et al. 2014) find a relatively tight relationship between the stellar mass and star formation rate (SFR) of star-forming galaxies (the so-called star-forming "main sequence"), but according to the hierarchical growth paradigm, increased merger rates should result in a much weaker correlation of these two quantities at high redshift. Observing this transition would pinpoint the end of the initial growth phase, providing strong constraints for models of early structure formation and the nature and causes of reionization.

This transition is currently not well constrained at $z \gtrsim 6$ due to the small number of galaxies with the necessary SFR and stellar mass estimates required, which only the combination of $H S T /$ WFC3 and Spitzer IRAC can provide at present. However, recent results using HST photometry (Bouwens et al. 2015, 2016; Davidzon et al. 2017) indicate that the galaxy luminosity function flattens between $z \sim 7$ (where there is likely an exponential cutoff) and $z \sim 10$ (where no cutoff is observed), consistent with the epoch around $z \sim 8-10$ that is the critical transition window.

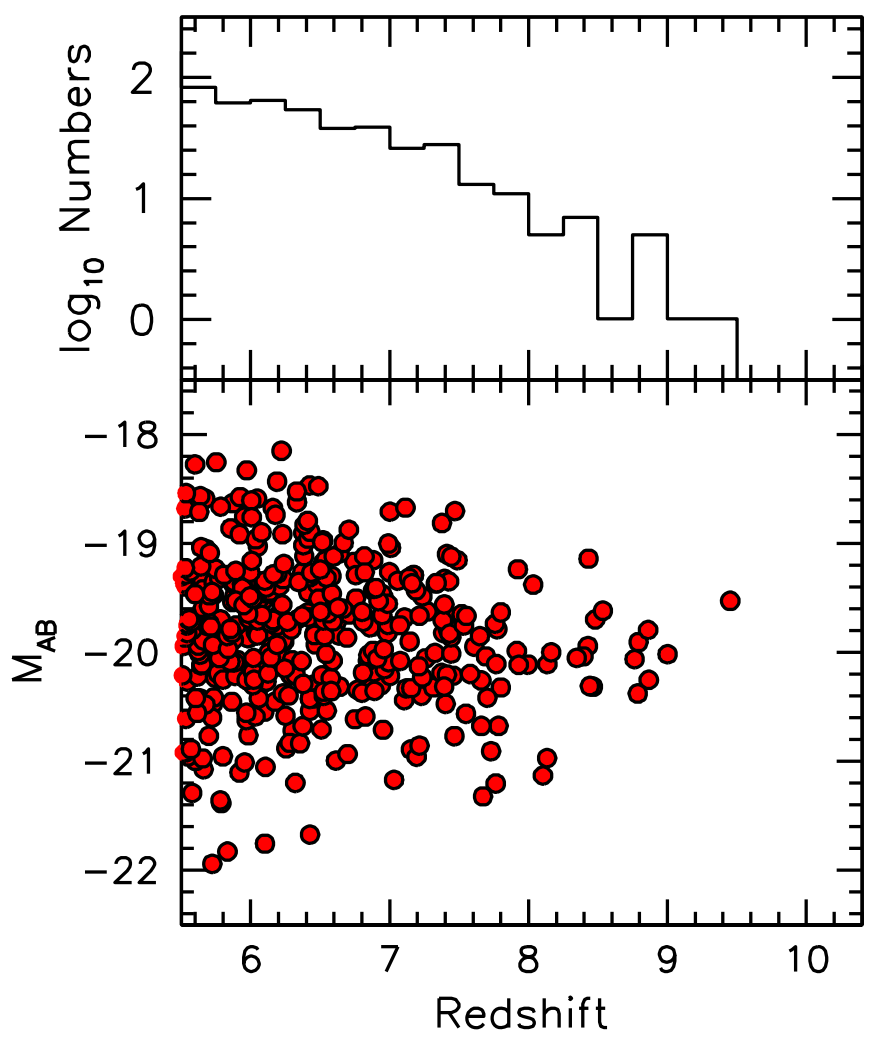

Figure 3. Simulated high-redshift, luminous galaxies (bottom) and overall distribution (top) in a random selection corresponding to the BUFFALO clusters and flanking fields. It is likely that the highest-redshift BUFFALO galaxy will lie at $z \sim 9-10$.

Uncertainties at this epoch are currently dominated by a combination of cosmic variance, insufficient area in current sight lines, and lack of ultradeep Spitzer data needed to confirm luminous $z>8$ systems. To illustrate the point, all published $z \sim 9-10$ CANDELS galaxies are in just two of the five possible fields where they could be discovered (Oesch et al. 2014; Roberts-Borsani et al. 2016). The BUFFALO survey is optimized to efficiently search for these early, high-mass galaxies over the remaining areas where mass estimation will be possible (Figure 3).

In addition to the expected change in the shape of the galaxy mass function, recent studies using HST and Spitzer observations (Lee et al. 2013; Stark et al. 2013; Steinhardt et al. 2014; Bouwens et al. 2015, 2016; Oesch et al. 2016) find a substantial population of galaxies that are seemingly too massive, too early to have formed through standard hierarchical merging (Steinhardt et al. 2016; Behroozi et al. 2019). However, these massive galaxies are also expected to be the most strongly biased, and thus the measurements are most affected by cosmic variance, and the factor of $\sim 2$ improvement in their measured number density provided by BUFFALO will either relieve or significantly increase this tension.

BUFFALO is designed to optimally reduce the current uncertainty by imaging sight lines with existing, sufficient ultradeep Spitzer data. Joint HST and Spitzer observations have proven to be essential for the discovery and characterization of the highest-redshift galaxies and recently resulted in the first rest-frame optical detection and stellar mass measurements of individual galaxies at $z \sim 8$ and even $z \sim 10$ (Labbé et al. 2013; Oesch et al. 2013). 


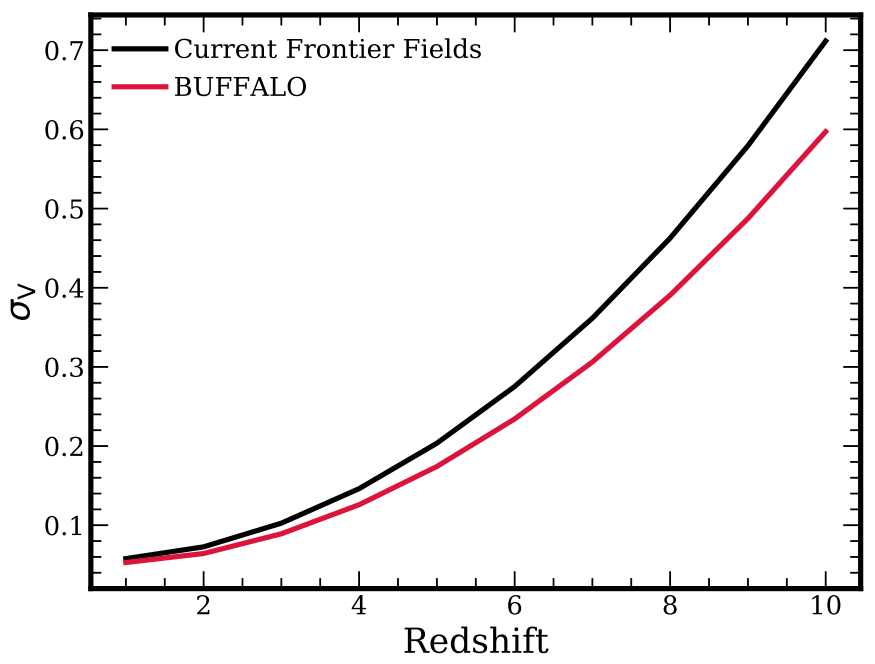

Figure 4. Predicted cosmic variance $\sigma_{v}$ for galaxies at stellar mass $M_{*} \sim 10^{9} M_{\odot}$ as a function of mass and redshift. Values are calculated using the prescription given in Moster et al. (2011), extrapolated to higher redshift as required.

Because early massive galaxies are very highly biased (Moster et al. 2011; see also Figure 4), the limiting factor in our understanding of this transition period is a combination of cosmic variance and area coverage. Of the three $z=10$ galaxy candidates in $\sim 800 \mathrm{arcmin}^{2}$ of deep HST imaging over the five CANDELS sight lines, two lie in a single $4 \operatorname{arcmin}^{2}$ region (Oesch et al. 2014), and the $z>7$ candidates in the HFF fields are highly clustered. The addition of BUFFALO data will improve the measurement of the space density of $L>L_{*}$ galaxies by doubling the number of sight lines with sufficient area, filter coverage, and Spitzer data to reliably detect galaxies at $z \sim 8-10$.

Spitzer data are essential for $z>8$ galaxy studies (see Bradač et al. 2014, Figure 3) because they constrain the age and mass of high-redshift galaxies and can be used to effectively remove $z<3$ contaminants. Spitzer has already invested $\sim 1500 \mathrm{hr}$ ( 2 months) imaging the HFF areas to $50-75 \mathrm{hr}$ depth, but HST WFC3-IR data exist over only $\lesssim 10 \%$ of this deep Spitzer coverage. The BUFFALO pointings were chosen to overlap with the existing Spitzer HFF coverage as efficiently as possible in regions central enough that there is still likely to be nonnegligible magnification from weak lensing. The BUFFALO coverage area also expands WFC3 coverage to an area that is well matched with the James Webb Space Telescope (JWST) NIRSPEC field of view.

BUFFALO will complete the WFC3-IR and ACS coverage of areas with Spitzer IRAC deep enough to study the high- $z$ universe over a large area, resulting in the best study of this key period currently possible. Critically, the large area and additional sight lines will both mitigate the effect of cosmic variance due to the strong clustering of $z>8$ objects (Trenti et al. 2012; Oesch et al. 2013; Laporte et al. 2014; Zheng et al. 2014) and directly constrain the galaxy bias with respect to the dark matter (Adelberger et al. 1998; Robertson et al. 2010).

Presently, Spitzer IRAC data at depths greater than $50 \mathrm{hr}$ pixel $^{-1}$ in both the 3.6 and $4.5 \mu \mathrm{m}$ bands are the only way to measure the stellar masses of galaxies at $z \sim 8-10$ and firmly establish that they are indeed high-redshift (e.g., Figure 5), while HST data are crucial to identifying candidate $z \sim 8-10$ galaxies through their photometric dropout. This powerful combination of data currently exists over $<740 \operatorname{arcmin}^{2}$ of sky, yielding $\sim 20$ joint detections of $z \sim 8$ galaxies and $\sim$ four at $z \sim 10$. The strong clustering of high-redshift galaxies has been definitively observed, with the majority of $z \sim 10$ galaxies found in one of five CANDELS fields (Bouwens et al. 2015) and one of the Frontier Fields known to contain an overdensity of $z \sim 8$ galaxies in the region mapped by BUFFALO (Zheng et al. 2014; Ishigaki et al. 2015).

\subsection{Clustering as a Test of Hierarchical Merging}

The small volume probed by previous Frontier Field observations at $z \sim 8$ results in a very large expected fieldto-field variance of $40 \%-50 \%$, currently dominated by Poisson error in each field at the bright end. This significantly limits the ability of the HFFs to measure accurate cosmic average quantities and true cosmic variance (Robertson et al. 2014). The additional coverage from BUFFALO will reduce this fieldto-field variance by $\sim 2 \times$, allowing an improved measurement of the true cosmic variance.

The true variance in number counts per field provides a direct and simple measure of the galaxy bias at these redshifts, allowing us to estimate the dark matter halo masses hosting these galaxies (e.g., Adelberger et al. 1998; Robertson et al. 2010). The availability of combined $H S T+$ Spitzer imaging will further allow us to determine the galaxy bias as a function of stellar mass-a crucial test of theoretical models (Behroozi \& Silk 2015; O'Shea et al. 2015). Similar tests are not possible in the significantly more numerous BoRG (Trenti et al. 2012) fields because they lack the ancillary Spitzer data required to measure stellar masses of high-redshift galaxies, although follow-up Spitzer observations have been conducted in some fields with high-redshift candidates (Morishita et al. 2018; Bridge et al. 2019).

\subsection{Improvement in Magnification Estimates}

As discussed in Section 3, a lack of knowledge of the presence of substructures in the outskirts of clusters can lead to underestimation of the mass and thus the density profile of the cluster by up to $20 \%$ (Acebron et al. 2017). Magnification at a given location in the cluster is a direct product of the mass estimate (and the redshift of a background source). Therefore, the expanded BUFFALO observing area provides a unique data set that can overcome the lack of accuracy of core-only mass models by being able to precisely detect where substructures are located and estimate their mass (down to a few percent precision thanks to $H S T$ resolution; Jauzac et al. 2015b). The exact improvement in magnification estimates with BUFFALO is difficult to predict, as it strongly depends on the dynamical state of each cluster, i.e., how many substructures exist and how massive those substructures are. However, once all BUFFALO clusters are analyzed, we will have for the first time a "statistical" sample allowing us to precisely estimate the bias induced by substructures on the magnification, which can then be related to the mass of those substructures.

\section{Structure Evolution and Dark Matter Physics}

The BUFFALO survey offers an unprecedented look at the large-scale structure of our universe by providing highresolution observations from space with $H S T$ over an unusually wide area around the six massive Frontier Fields clusters. Flanking the core of those clusters with several pointings has extended the field of view out to $\sim 3 / 4$ of the virial radius. This 

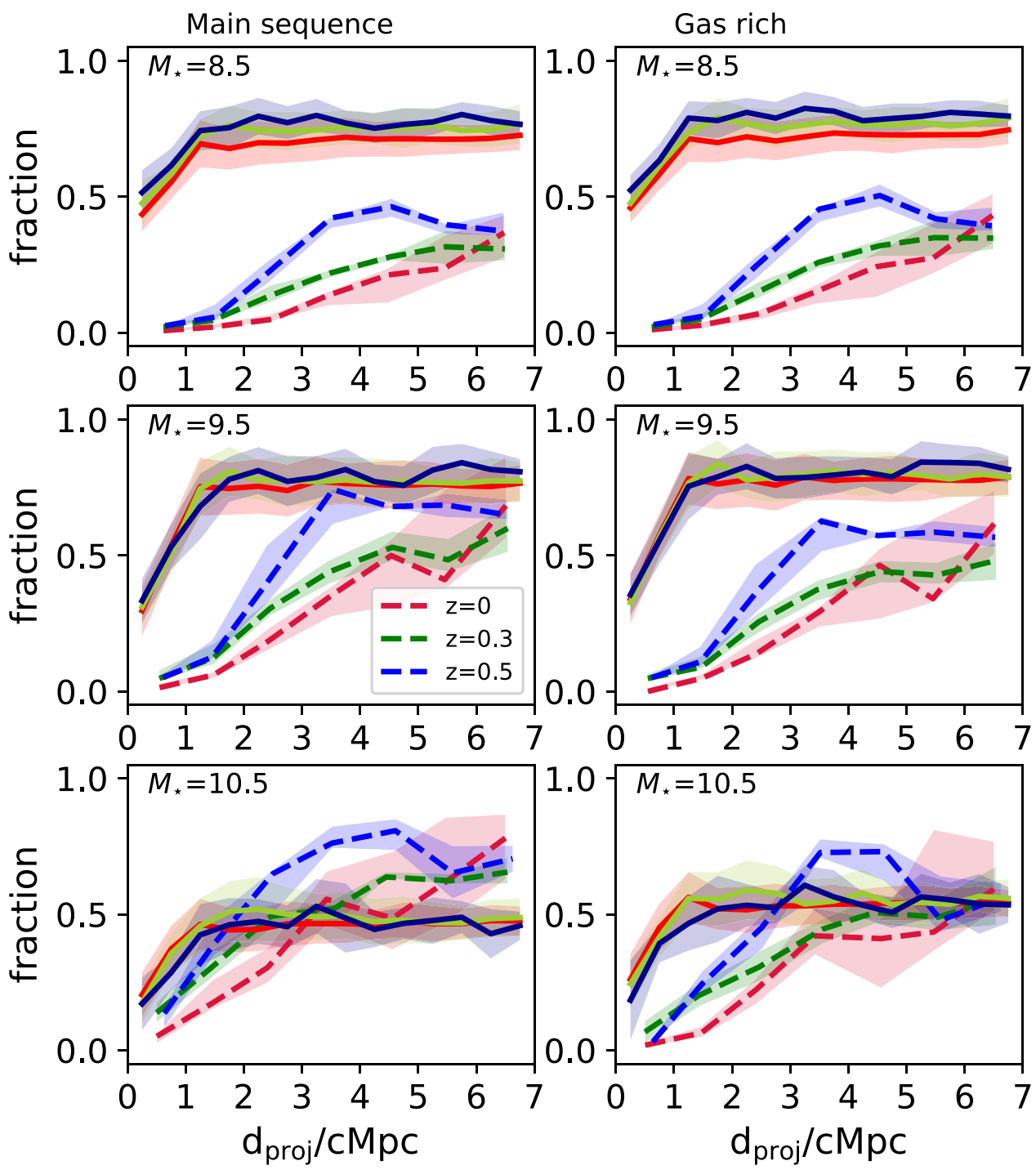

Figure 5. Fraction of star-forming (left) and gas-rich (right) galaxies as a function of clustercentric distance at three redshifts, $z=0,0.3$, and 0.5 (red, green, and blue solid lines, respectively), and three stellar mass bins, as labeled, in clusters of mass $M_{200 \mathrm{c}}>5 \times 10^{14} M_{\odot}$ in the cosmological semianalytic model of galaxy formation SHARK (Lagos et al. 2018; solid lines) and the cluster hydrodynamical simulation zooms C-EAGLE (Bahé et al. 2017; Barnes et al. 2017; dashed lines). For both simulations, we show the medians and 25th-75th percentile ranges (thick lines with shaded regions, respectively). Star-forming and gas-rich galaxies are defined as those with sSFR/MS $\left(M_{\text {star }}\right)>0.25$ and $f_{\text {neutral }} /\left\langle f\left(M_{\text {star }}\right)\right\rangle>0.25$, respectively. Here $\mathrm{MS}\left(M_{\text {star }}\right)$ and $f\left(M_{\text {star }}\right)$ are the main-sequence sSFR and median $f_{\text {neutral }}$ of mainsequence galaxies at $M_{\text {star }}$, respectively, and $f_{\text {neutral }}=\left(M_{\mathrm{HI}}+M_{\mathrm{H}_{2}}\right) / M_{\text {star }}$.

significant improvement is designed to allow improved investigations into the origin and evolution of large-scale structure. The current consensus model, $\Lambda \mathrm{CDM}$, assumes the hierarchical evolution of structures. They grow at a robustly predicted rate, fed by the cosmic web's filaments, which carry dark matter and baryons toward the centers of what will become large clusters (Bond et al. 1996).

However, so far, very few observations have been able to detect those large-scale filaments (Dietrich et al. 2012; Jauzac et al. 2012; Eckert et al. 2015; Connor et al. 2018). BUFFALO now allows an unprecedented resolution for mapping of dark matter and will deliver data to detect material (both baryons and dark matter) falling on the clusters by the direct detection of galaxies and enhanced star-forming regions as tracers of the filaments and indirect detection of dark matter densities using weak lensing. Clearly identifying and locating individual filaments and the local cosmic web could be useful in constraining their effect on star formation and quenching (Aragon Calvo et al. 2019), galaxy angular momentum (Goh et al. 2019), and even, through a "spiderweb test" (Neyrinck et al. 2018), redshift-to-distance mapping.

In addition, reaching further away from the cluster center at the $H S T$ resolution will sharpen our understanding of merging events. Indeed, all six HFF clusters show various states of merging (see Lotz et al. 2017 and references therein.) The extended view of the clusters can now be used to map the underlying dark matter distribution using both strong and weak lensing. At the depth and width of existing Frontier Fields observations, strong lensing already revealed a detailed view of the dark matter distribution in the cluster cores, but it is known that the mass in the cluster outskirts can influence the inner core profile (Acebron et al. 2017; Mahler et al. 2018). BUFFALO now provides a unique view of the global mass distribution of clusters up to $\sim 3 / 4 \times R_{\text {vir }}$.

A few percent accuracy on weak lensing measurements will be reachable due to highly constrained photometric redshift selection, primarily because of the unique HST and Spitzer/ IRAC coverage of the BUFFALO fields. Extensive 
spectroscopic follow-up of these clusters is planned as well. The broader lensing mapping of the substructures will break the degeneracy coming from the outskirt mass contribution to the lensing potential.

The $\Lambda$ CDM and hierarchical merging also predict the evolution of galaxies while falling in clusters. Extending $H S T$ observations to $\sim 3 / 4 R_{\text {vir }}$ also provides stringent observational constraints for the study of galaxy evolution within dense environments.

Niemiec et al. (2019) studied and quantified dark matter stripping of galaxy halos during their infall into clusters, as well as the evolution of their stellar mass and star formation using the Illustris-1 simulation. Such studies are often made in aggregate on a large number of clusters, but BUFFALO will do it on a cluster-by-cluster basis, avoiding smoothing out smallerscale influences on individual clusters. This also allows insight into the stripping of dark matter halos; environmental quenching mechanisms, including galaxy-galaxy interactions and harassment (Moore et al. 1996); strangulation (Balogh et al. 2000; Peng et al. 2015); the evolution of the stellar population; and the galaxy gas reservoir, providing critical insight into the cluster assembly history. The infall regions and intermediate-density environments traced by BUFFALO provide ideal observations to study these interactions (Moss 2006; Perez et al. 2009; Tonnesen \& Cen 2012).

Cen et al. (2014) reported a steep increase in the fraction of star-forming galaxies from the cluster center up to $2 \times R_{\text {vir }}$. There is little consensus on this, as is shown in Figure 5 for two galaxy formation simulations, the semianalytic model SHARK (Lagos et al. 2018) and the cosmological hydrodynamical simulations cluster suite C-EAGLE (Bahé et al. 2017; Barnes et al. 2017). There are many differences worth highlighting: the simulations predict different star-forming/gas-rich galaxy overall fractions, particularly at low stellar masses $\left(<10^{9} M_{\odot}\right)$; C-EAGLE predicts fractions that continue to rise even out to 3-4 $\times R_{\text {vir }}$, while SHARK predicts a steep increase out to $1-1.5 \times R_{\mathrm{vir}}$, followed by a flattening; and C-EAGLE predicts much stronger evolution over the last 5 billion yr than SHARK. This shows that the observations of BUFFALO, combined with local universe cluster observations, will offer strong constraints on the models, hopefully allowing one to rule out some of the wildly different behavior seen in Figure 5. Note that these differences arise even though both simulations account for quenching mechanisms typical of galaxy clusters, such as ram pressure stripping and lack of cosmological accretion, among others (Gunn et al. 1972; Bahé et al. 2013). Figure 5 also shows the fraction of gas-rich galaxies, computed from their atomic plus molecular gas fraction, as a proxy for good candidates of "jellyfish" galaxies. Poggianti et al. (2019) showed that the jellyfish galaxy population can be used to investigate the density of the cluster gas halos by probing the material in starforming regions coming out of the galaxies as they fall into the cluster. In BUFFALO, we expect to probe these galaxies at intermediate redshifts, building on previous studies in HFF clusters (Schmidt et al. 2014; Treu et al. 2015; Vulcani et al. 2016).

In recent decades, the theoretical framework that describes the formation of cosmic structures has been tested by increasingly precise observations (Planck Collaboration et al. 2016a), which show good agreement with several key aspects of current models. BUFFALO offers an orthogonal probe by providing a detailed view of the clustering, as well as its synergy with simulations of the universe and massive clusters such as the HFF ones. The Frontier Fields clusters remain rare massive clusters in simulations (Jauzac et al. 2016a, 2018), and a better understanding of their environment, only possible with BUFFALO, will shed light on how they form and evolve. By finding simulated analogs of those six clusters, it is also possible to test various related cosmological effects on the growth rate, mass ratio among substructures, and merging events.

\subsection{Cluster Science and Dark Matter Physics}

High-resolution space-based observations of galaxy clusters have revolutionized the study of dark matter (Natarajan et al. 2002; Limousin et al. 2007; Richard et al. 2010). Early observations of merging galaxy clusters presented some of the most conclusive and unequivocal evidence for the existence of dark matter, while placing stringent limits on modified theories of gravity. For example, the Bullet Cluster, a postmerger collision of two galaxy clusters in the plane of the sky, clearly shows the separation of intracluster gas and its associated cluster members (Clowe et al. 2004; Bradač et al. 2006). Thanks to weak lensing, it was found that the majority of the mass lies in the galaxies, not the gas, as suggested by modified gravity theories (Clowe et al. 2006). Following this study, multiple other merging clusters were found to exhibit similar properties, and detections of such offsets soon became ubiquitous (Bradač et al. 2008; Merten et al. 2011; Harvey et al. 2015; Jauzac et al. 2016a).

As a result of these studies, it soon became clear that merging clusters could provide further insights into dark matter and not just evidence for its existence. For example, it is possible to constrain the self-interaction cross section of dark matter using these clusters (Harvey et al. 2013; Kahlhoefer et al. 2014; Robertson et al. 2019). Self-interacting dark matter (SIDM) is an extension of the cold and collisionless dark matter paradigm that has received a lot of interest in the last decade. With its discriminative signals, tests of SIDM models provide a unique window into the physics of the dark sector.

With the discovery of multiple colliding systems, it soon became possible to collate these events and statistically average them in order to provide further constraints on SIDM. Methods that estimated the relative positions of dark matter, galaxies, and gas were developed to statistically average over many merging events (Massey et al. 2011; Harvey et al. 2014). The first study of 30 merging galaxy clusters was carried out by Harvey et al. (2015) and constrained the cross section of dark matter to $\sigma_{\mathrm{DM}} / m<0.5 \mathrm{~cm}^{2} \mathrm{~g}^{-1}$. However, a subsequent study found potential systematic uncertainties and revised this estimate to $\sigma_{\mathrm{DM}} / m<2 \mathrm{~cm}^{2} \mathrm{~g}^{-1}$ (Wittman et al. 2018). Either way, it was clear that there was statistical potential in these merging systems.

The BUFFALO survey will extend the already successful HFF program, providing a unique insight into the dynamics of dark matter during halo infall. Probing the regions out to $\sim 3 / 4 r_{\text {vir }}$, BUFFALO will examine a regime where the unknowns of core passage will be circumvented, and positional estimates of the substructures will be cleaner.

Indeed, HST high resolution will allow us to precisely locate and weigh dark matter substructures in the clusters (down to the percent level precision on mass measurement and $6 \%$ on the location of dark matter peaks; Jauzac et al. 2014, 2015b). It will then be possible to trace their dynamical history by combining 
the lensing analysis with the plethora of multiwavelength data available on these six clusters; e.g., X-rays will help trace the gas, while optical, near-infrared, and UV imaging and spectroscopy will help understand the dynamics and kinematics of the galaxies in the detected substructures. Moreover, with sample-specific simulations of multiple dark matter models, it will be possible to monitor, test, and mitigate all known and unknown systematic errors. As a result, BUFFALO will provide the cleanest measure of SIDM from infalling substructures to date.

In addition to monitoring the trajectories of infalling halos to constrain SIDM, understanding the mass function within the clusters will provide important insights into the dynamics of dark matter. For example, it has been suggested that A2744 exhibits too much substructure (Jauzac et al. 2016a; Schwinn et al. 2017) when compared to the predictions of standard CDM. This finding could possibly be an indication of exotic dark matter physics; however, in such current small fields of view and on one sample, it is difficult to establish statistical significance. The extended imaging of BUFFALO with novel methods to compare observations to simulations, such as the cluster power spectrum (Mohammed et al. 2016) and peak analysis (Fan et al. 2010), will provide important insights into the substructure mass function of clusters and the dark matter that drives these statistics.

\subsection{D Halo Structure and Nonthermal Pressure Support}

How the dark and baryonic masses distribute in the halo's gravitational potential is a fundamental prediction of models of large-scale structure formation, allowing to use galaxy clusters as astrophysical laboratories, cosmological probes, and tests for fundamental physics. Probes at different wavelengths (optical, Sunyaev-Zeldovich (SZ), and X-ray) can be used and combined to reconstruct 3D triaxial ellipses describing the geometrical shape of the gas and total mass distribution, as obtained from the CLUster Multi-Probes in Three Dimensions (CLUMP-3D) project on $16 \mathrm{X}$-ray regular CLASH clusters (Chiu et al. 2018; Sereno et al. 2018; Umetsu et al. 2018). In this analysis, a weak lensing signal constrains the 2D mass and concentration, which are deprojected thanks to the information on shape and orientation from the X-ray (surface brightness and temperature) and SZ. The mass and concentration can then be determined together with the intrinsic shape and equilibrium status of the cluster as required by precision astronomy through a Bayesian inference method and not relying on the assumptions of spherical symmetry or hydrostatic equilibrium, which could bias results. The joint exploitation of different data sets improves the statistical accuracy and enables us to measure the 3D shape of the cluster's halo and any hydrostatic bias, evaluating the role of the nonthermal pressure support. In general, they obtained that the shapes are in good agreement with the predictions from the standard $\Lambda \mathrm{CDM}$ cosmological model. However, compared to simulations, the data show a slight preference for more extreme minor-to-major axial ratios. We need a combination of more sensitive observational data to probe, also as a function of halo mass and dynamical state, the 3D structure of the gas and dark matter distribution, assessing their consistency with $\Lambda \mathrm{CDM}$ predictions and their equilibrium once geometrical biases (like projection) are corrected for.

\subsection{Galaxy Evolution}

As the largest observable gravitationally bound structures in the universe, galaxy clusters provide a unique tool for exploring the coeval evolution of galaxies and cosmic structures. Very effective star formation quenching is observed in clusters at all redshifts, where the fraction of star-forming galaxies is lower than in the field (Hashimoto et al. 1998), and the fraction of early-type morphologies (lenticulars, ellipticals) is the highest (Dressler 1980). A major thrust of ongoing research is to understand these transitions, particularly using deep HST imaging of clusters (Martinet et al. 2017; Wagner et al. 2017; Marian et al. 2018; Olave-Rojas et al. 2018; Connor et al. 2019; Rodríguez-Muñoz et al. 2019).

There are strong hints that star formation suppression already occurs at large distances from the cluster cores (Bahé \& McCarthy 2015; Haines et al. 2015), and that red galaxies are located preferentially close to filament axes (e.g., Malavasi et al. 2017; Laigle et al. 2018). Therefore, understanding the preprocessing of galaxies requires studies that move to regions well beyond the cluster core. Color gradients are required to unveil the location of recent star formation (Villalobos et al. 2012; Liu et al. 2018), as well as evidence for stripping and quenching, e.g., so-called "jellyfish" galaxies in the process of being ram pressure stripped (e.g., in A2744; see Owers et al. 2012).

The BUFFALO survey is perfectly suited for these studies, as it traces the intermediate-density environments where the quenching processes occur. The multiwavelength data provide high-resolution measurements of the colors, SFRs, morphologies, and local environments of galaxies extending out to the edges of the massive HFF clusters, enhancing the legacy value of the Frontier Fields observations.

\subsection{Intracluster Light}

One of the most intriguing signatures of the assembly of galaxy clusters is a diffuse light known as ICL. This light is composed of a substantial fraction of stars not gravitationally bound to any particular galaxy but to the cluster potential. Observations have shown that the ICL is the product of interactions among the galaxies in the cluster. In this sense, the ICL is a unique tracer of how the assembly of the cluster proceeds through cosmic time (see Montes 2019, for a review).

The integrated stellar population properties revealed by the ICL tell us about the dominant process responsible for the formation of this diffuse light and therefore of the mechanisms at play in the assembly of the cluster. Different scenarios for the origin of the ICL result in different stellar population properties, ranging from the shredding of dwarf galaxies (Purcell et al. 2007; Contini et al. 2014) to violent mergers with the central galaxies of the cluster (Conroy et al. 2007; Murante et al. 2007), in situ formation (Puchwein et al. 2010), or the preprocessing of the diffuse light of groups infalling into the cluster (Mihos 2004; De Lucia et al. 2012). These different mechanisms might vary within the cluster and during the history of the cluster (Cañas et al. 2019b).

Several works have already studied the ICL in the HFF clusters (Montes \& Trujillo 2014, 2018; Jiménez-Teja \& Dupke 2016; Morishita et al. 2017), but one of the difficulties encountered in these studies is the limited field of view of the HFF observations that prevents accurate sky subtraction and, therefore, accurate properties of this light at large radius from 


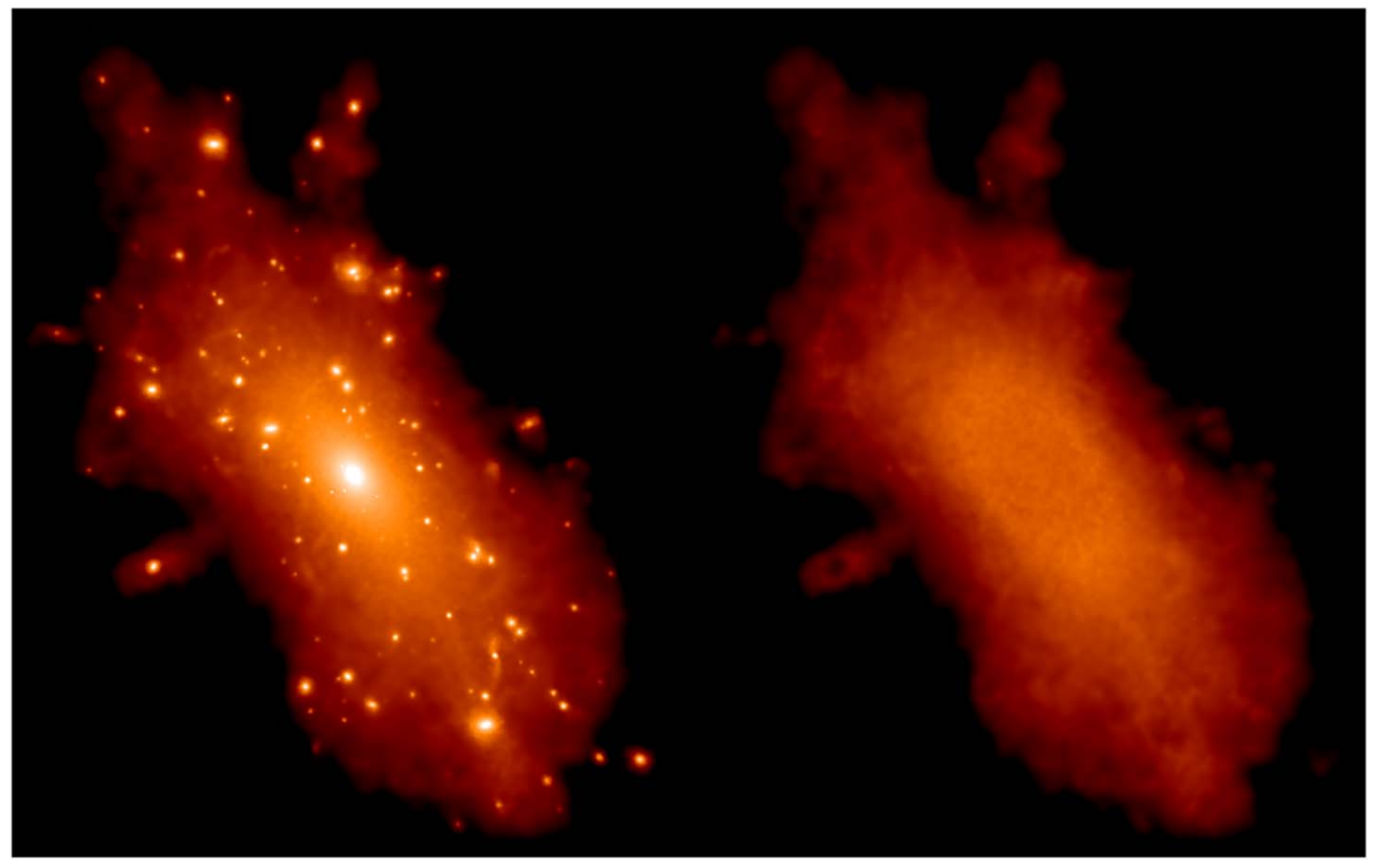

Figure 6. Example of a simulated massive cluster of $M_{200, \text { crit }} \approx 10^{15} M_{\odot}$ from the C-EAGLE simulation (Bahé et al. 2017; Barnes et al. 2017) at $z=0.3$. The left panel shows a stellar mass map of the whole 3D friends-of-friends region, while the right panel shows the ICL only, defined as the kinematically hot stellar component using the full 6D information available in cosmological simulations (see Cañas et al. 2019a for details of the algorithm and Cañas et al. 2019b for an analysis of the intrahalo stellar component of galaxies across environments and cosmic time). BUFFALO will allow us to measure the fraction of total light in the ICL and its stellar population properties to test the predictions above and investigate how this component forms.

the central parts of the clusters. These studies have found that the main mechanism to produce ICL is the tidal stripping of massive satellites $\left(\sim 10^{10-11} M_{\odot}\right)$. However, these results only describe the more central parts of the clusters $(<200 \mathrm{kpc})$.

Using BUFFALO, we will be able to explore the ICL in detail up to $\sim 3 / 4 R_{\text {vir }}$. That will allow us to explore the formation mechanisms at play at large cluster radius and expand our knowledge of the formation of this diffuse light. The amount of light in the ICL provides information on the efficiency of the interactions that form this component (see Figure 6). To date, simulations have provided contradictory predictions as to how the amount of ICL depends on halo mass, as well as the expected evolution with time, with some works finding no dependence on halo mass (e.g., Rudick et al. 2011; Contini et al. 2014), while some others report a clear dependence (e.g., Murante et al. 2007; Cui et al. 2014). Evolutionwise, some authors reported strong (e.g., Contini et al. 2014) or rather weak (e.g., Rudick et al. 2011) evolution of the ICL fraction. Some of these discrepancies are due to the variety of numerical definitions of ICL (see discussion in Cañas et al. 2019b), which generally use the 3D position of particles in simulations. Cañas et al. (2019a) introduced a full 6D method to define ICL and applied it to the Horizon-AGN simulations (Cañas et al. 2019b; see Figure 6) and C-EAGLE (R. Cañas et al. 2020, in preparation) to show that the ICL fraction is remarkably flat at the galaxy cluster regime and that different simulations agree once the same ICL definition is applied. BUFFALO will place unique constraints on the ICL fraction, which, together with local universe measurements, will provide a large cosmic time baseline to compare with simulation predictions. The multiwavelength coverage of BUFFALO is crucial to derive the properties of the stellar populations of the ICL within the cluster to study how its different formation mechanisms vary with clustercentric distance. We will also be able to measure diffuse light in substructures and quantify for the first time the amount of light that will end up as ICL through preprocessing.

Finally, recent work has demonstrated that the ICL accurately follows the shape of the underlying dark matter halo in clusters of galaxies (Montes \& Trujillo 2019). This results was only possible thanks to the superb mass modeling available for the HFF clusters. The next step will be to extend this analysis to larger scales to assess whether the similarity between the distributions of ICL and mass holds at a larger cluster radius. Simulations show that this similarity should hold out to $1.1 \mathrm{Mpc}$ (I. Alonso-Asensio et al. 2020, in preparation), and with BUFFALO, we will be able to finally assess it.

One limiting factor for deriving ICL brightness profiles is establishing a sky background. The outskirts of the HFF primary field still contain contributions from ICL, but observations in the same filters in the parallel fields were taken at different times. Due to the temporal variations of the sky background, the parallel fields cannot be used by 


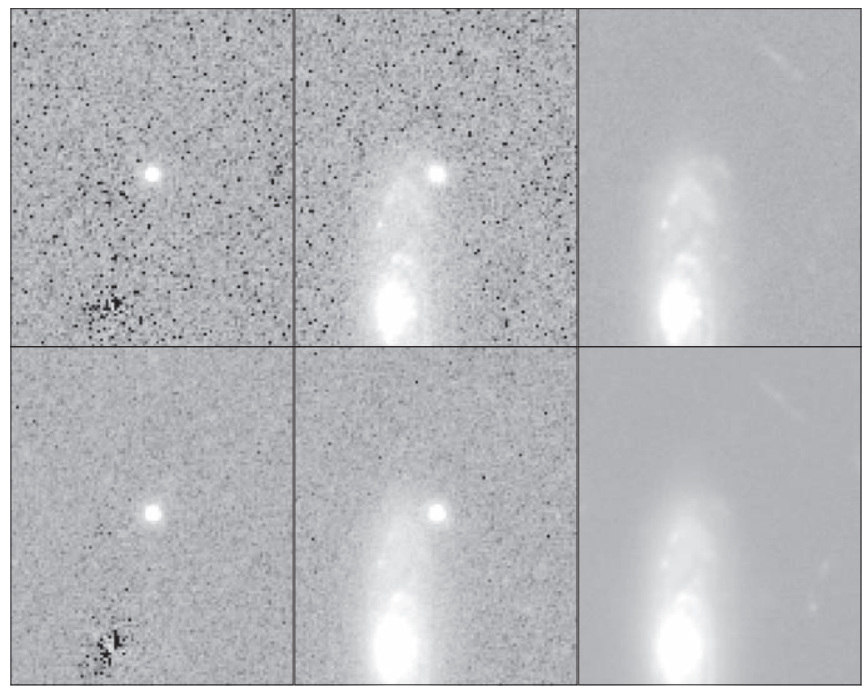

Figure 7. Example of $\mathrm{SNe}$ detected in the BUFFALO fields at R.A. 22:49:13.700, decl. $-44: 32: 38.736$. From right to left are $6^{\prime \prime}$ cutouts of the reference HFF image, the BUFFALO epoch 1 image, and the difference image. The top row shows the F606W filter, and the bottom row shows the F814W filter.

themselves to calibrate a sky background for use in the cluster center (DeMaio et al. 2015).

Thus, in addition to the previously described observations, BUFFALO data of MACS J1149 also include six additional orbits of imaging taken by GO-15308 (PI: A. Gonzalez). These six orbits were divided into three pointings of two orbits each; each pointing was observed with the F105W filter and the F160W filter on WFC3/IR for one orbit each. The three pointings linearly bridge the primary and parallel HFF fields such that each pointing has a $20 \%$ spatial overlap with its neighbors. Due to this overlap, temporal variations can be accounted for, and the dominant uncertainty in sky background becomes that of the flat-fielding process. Based on work by DeMaio et al. (2018), it is expected that the flat-fielding should reduce the residual systematic uncertainty to $\mu>31$ mag arcsec$^{-1}$, enabling a measurement of the ICL radial brightness profile down to $\mu_{160}=29.5 \mathrm{mag} \operatorname{arcsec}^{-1}$. In addition, GO-15308 includes parallel ACS observations in F606W and F814W, which extend the coverage of MACS J1149 in three pointings per filter to the side of the cluster opposite the HFF parallel field.

\section{SNe and Other Transients}

The observations of this program have been purposely spaced into two visits (or epochs) per pointing to allow for the discovery of $\mathrm{SNe}$ and other transients. By design, revisits to the same field in the same filter sets are separated by approximately 30 days, approximately matching the rise time of most SN types, including SNe Ia, around $z=1$. We expect this to yield a discovery rate per visit that is comparable to that in the CLASH (Postman et al. 2012), which will amount to about 10-25 events over the full duration of the BUFFALO survey.

An example of one such discovery is given in Figure 7, a transient discovered in the Abell S1063 difference imaging at $22: 49: 13.70,-44: 32: 38.74$. The cutouts show a $6^{\prime \prime}$ box around the region, showing the reference HFF image, the first-epoch BUFFALO observation, and the difference frame in which the search for SNe was conducted. This event is visible in both the

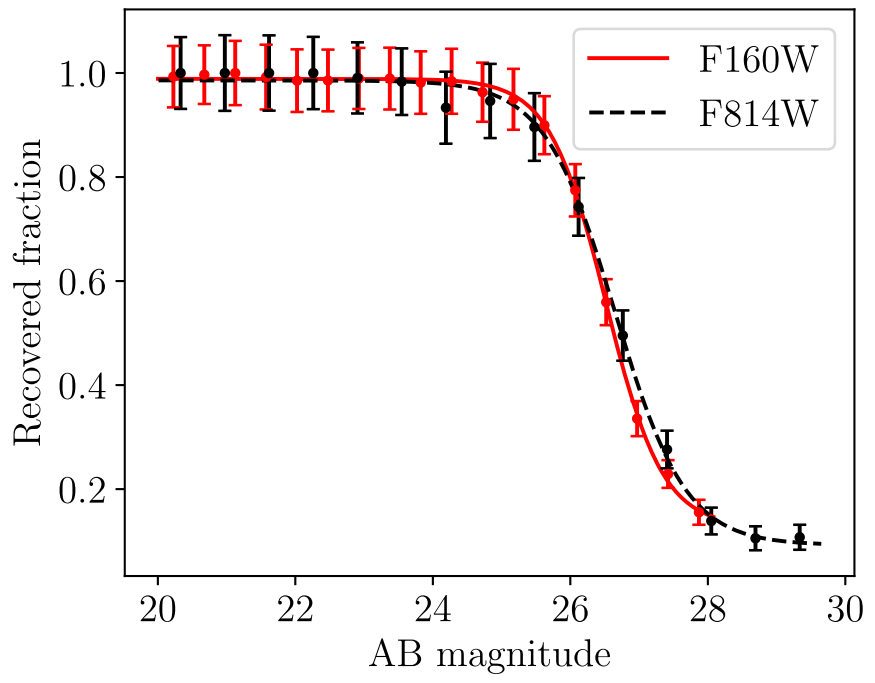

Figure 8. Expected efficiency of SN searches within the BUFFALO images. The recovered fraction of fake sources injected into BUFFALO difference images as a function of magnitude for the two reddest filters of the two HST cameras-F814W for ACS (black dashed line) and F160W for WFC3/IR (red solid line) - is shown. Sources are recovered if the peak pixel count rate of the source is above 0.03 counts $\mathrm{s}^{-1}$. The $50 \%$ recovery rate is 26.5 mag for both filters.

ACS observations of the parallel field, taken 2019 April and May, and the first epoch of the WFC3 parallel pointing, taken 2019 October, highlighting the power of the various time delays between BUFFALO images in the search for transient events.

A summary of the preliminary searches of each field, conducted after each epoch was processed, for $\mathrm{SNe}$ and transients is shown in Table 2. As of 22 October, 21 of the 28 camera-pointing combinations had been conducted, and thus the preliminary findings of six SNe suggest a total of eight over the entire BUFFALO program, slightly lower than predicted by CLASH. However, more thorough follow-up searches will be conducted that may yield fainter or more obscure detections, increasing the detection rate.

To probe the expected sensitivity of the BUFFALO difference images, we conducted a simple test. Fake sources were injected randomly into the drizzled difference images, with $\mathrm{AB}$ magnitudes in the range from 20 to $\sim 30$, with appropriate Poisson noise. These were then recovered using a simple peak finding algorithm, looking for sources with peak count rates above 0.03 counts $\mathrm{s}^{-1}$, roughly the lowest pixel count rate that is qualitatively visible above the BUFFALO difference images background by eye. The recovery fraction as a function of magnitude is shown in Figure 8. Given the similar zero-points of the two filters, the $50 \%$ recovery rate is $26.5 \mathrm{mag}$, and the $\sim 95 \%$ recovery rate is $\sim 25$ th magnitude for both. The very faintest recoverable $\mathrm{SNe}$ are $27 \mathrm{th}-28$ th magnitude in the F160W filter and 29th-30th magnitude in F814W, due in part to the differing detector pixel scales, drizzled onto a common 0 ". 06 scale. However, it should be noted that this efficiency rate is quite optimistic, as it requires a fairly quiescent background in both frames contributing to the difference image. High background count rates, or SNe lying near the center of galaxies, will suffer higher backgrounds or poor dither pattern noise (see left panels of Figure 7), raising the minimum count rate at which transients are detectable. Therefore, a more conservative estimate might put the minimum count rate at 0.3 counts $\mathrm{s}^{-1}$ and thus reduce the $50 \%$ completeness limit of detections to 24th magnitude and the $95 \%$ recovery rate to $22.5 \mathrm{mag}$. As all currently detected sources are of 


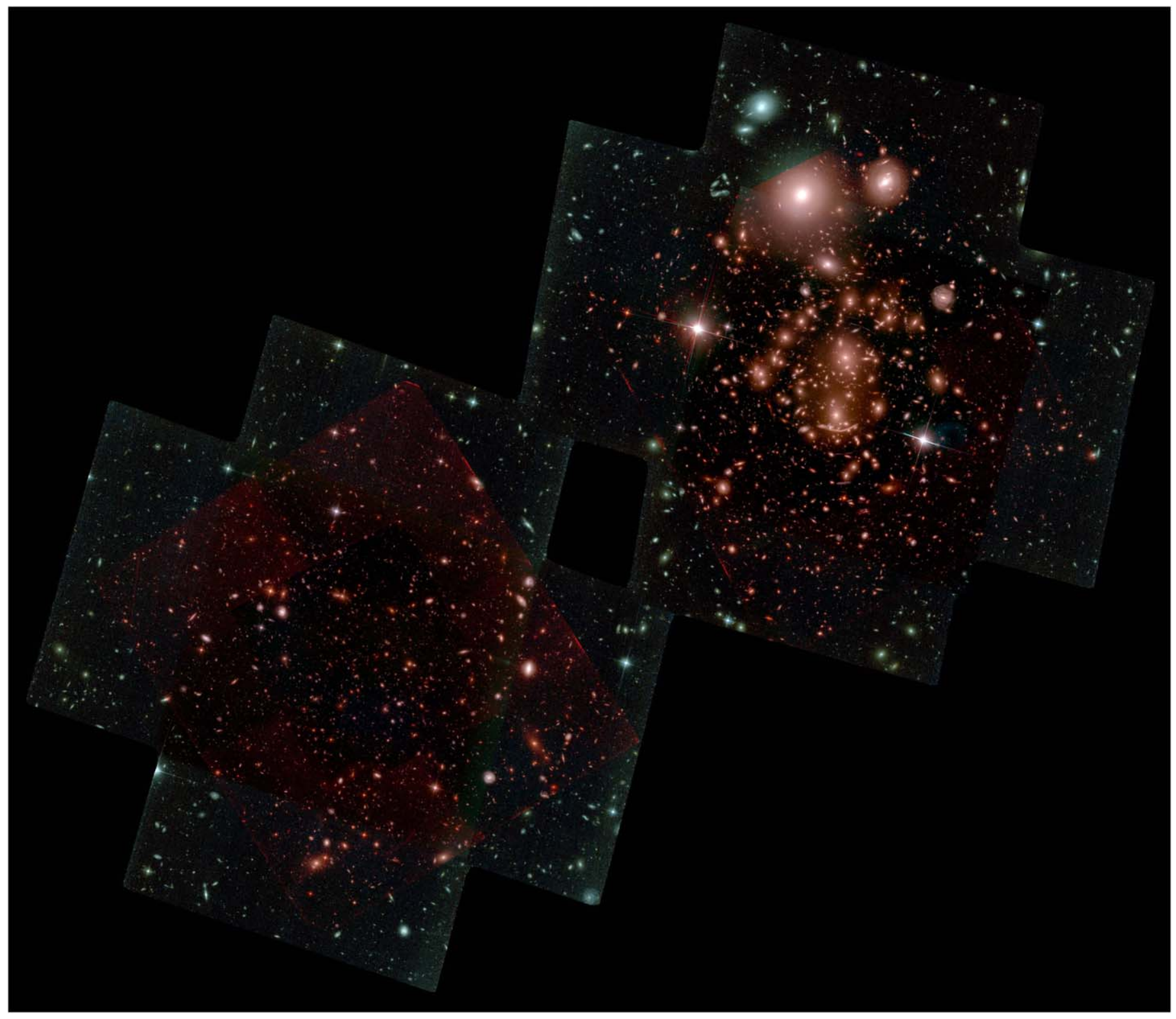

Figure 9. BUFFALO composite color image of A370. The BUFFALO field of view is four times larger than the previous Frontier Fields coverage (shaded in the central region for both the cluster and parallel fields), in addition to increasing the depth in the central region.

order 21st-22nd magnitude (see Table 2), it is likely that we are complete at this brighter detection threshold but will require followup studies to recover the very faintest objects.

The BUFFALO observations may contribute SN detections at a uniquely high redshift range. Infrared imaging programs with HST like this one have been the most efficient approach for discovery of SNe at $1.5<z<2.5$, the highest-redshift regime where $\mathrm{SNe}$ Ia and normal luminosity core-collapse $\mathrm{SNe}$ (CCSNe) have been detected (Graur et al. 2014; Rodney et al. 2014, 2015b; Strolger et al. 2015). Gravitational lensing from the clusters that dominate the center of each BUFFALO field will magnify background $\mathrm{SNe}$, making it possible to detect $\mathrm{SNe}$ at redshifts $2<z<3$ that would normally be undetectable (see Rubin et al. 2018), though lensing does reduce the high- $z$ survey volume behind the cluster (Barbary et al. 2012).

The BUFFALO program also could potentially locate $\mathrm{SNe}$ behind the clusters that are significantly magnified by gravitational lensing (Nordin et al. 2014; Patel et al. 2014; Rodney et al. 2015a). These lens-magnified events can provide a valuable test of the gravitational lensing models and have been informative in the refinement of lens models for Frontier Fields clusters. There is also a small but nonzero chance of locating another strongly lensed SN with multiple images, similar to "SN Refsdal" (Kelly et al. 2015). An SN that is multiply imaged by a cluster lens is likely to have measurable time delays (Kelly et al. 2016; Rodney et al. 2016) and could potentially be used to measure the Hubble constant (Grillo et al. 2018). Deep imaging surveys of the Frontier Fields clusters have revealed other exotic transient events with extreme magnifications $\mu>100$ (Rodney et al. 2018) or even $\mu>1000$ (Kelly et al. 2018; Chen et al. 2019). The BUFFALO imaging could discover such events at redshifts $0.8 \lesssim z \lesssim 2$, but the rates of such extreme microlensing events are highly uncertain, and the BUFFALO cadence is not optimized for their discovery (see, 
Table 2

BUFFALO SN Candidates

\begin{tabular}{|c|c|c|c|c|c|c|c|c|}
\hline Field & R.A. (H:M:S) & Decl. (D:M:S) & Detected Filters & AB Mag & Date & Pointing & Epoch & Redshift \\
\hline \multirow[t]{2}{*}{ MACS 0416} & $04: 16: 14.25$ & $-24: 03: 41.16$ & F606W & $\sim 22-23$ & 2019 Feb & Main & Epoch 2 & $z \sim 0.3$ \\
\hline & 04:16:33.05 & $-24: 06: 44.66$ & F606W & & 2019 Sep & Parallel & Epoch 2 & \\
\hline \multirow[t]{4}{*}{ Abell S1063 } & $22: 48: 53.56$ & $-44: 31: 19.59$ & F105W & & 2019 Jun & Main & Epoch 2 & \\
\hline & 22:49:13.70 & $-44: 32: 38.74$ & F606W & $22.40 \pm 0.20$ & 2019 Jun & Parallel & Epoch 2 & \\
\hline & & & F814W & $21.80 \pm 0.20$ & & & & \\
\hline & & & F160W & $22.61 \pm 0.04$ & & & & \\
\hline \multirow[t]{4}{*}{ A2744 } & $00: 14: 28.55$ & $-30: 23: 33.98$ & F606W & & 2019 Jul & Main & Epoch 2 & $z \sim 0.2-0.3$ \\
\hline & & & F814W & & & & & \\
\hline & $0: 14: 26.56$ & $-30: 23: 44.17$ & F606W & & $2019 \mathrm{Jul}$ & Main & Epoch 2 & \\
\hline & & & F814W & & & & & \\
\hline
\end{tabular}

however, Kaurov et al. 2019, in which they predicted an interval between caustic transient events of $1 \mathrm{yr}$ ).

\section{Data Products}

A major goal of BUFFALO is to produce data products that can be used by the entire astronomical community as both stand-alone catalogs and in preparation for additional observations with the JWST. Release of these products will be via MAST. $^{85}$ In an effort to release initial data as quickly as possible, these catalogs will be released individually for each of the six Frontier Fields clusters and parallel fields rather than waiting for the entire program to be complete.

\subsection{High-level Mosaics}

BUFFALO will release a mosaic in each of the five HST bands, combining the new data with previous Frontier Fields observations in the same bands where they are available. The first of these mosaics, A370 (Figure 9), is now available on MAST concurrent with the publication of this paper. Mosaics for the other clusters will be released as available, with a strong effort to release as many as possible prior to relevant proposal deadlines. Although these first data will be quickly presented, it is anticipated that more significant scientific value will come from value-added, multiwavelength catalogs and associated models.

\subsection{Catalogs}

BUFFALO will produce several value-added catalogs, with an initial release for A370 and updates planned as additional clusters are completed. These catalogs will exploit BUFFALO photometric data along with all available multiwavelength observations from the $H S T$ archive. A list of existing data sets can be found in Table 3 .

It will also include ancillary data from near-IR surveys (Brammer et al. 2016; M. Nonino et al. 2020, in preparation ${ }^{86}$ ) and the Spitzer images that are a cornerstone of the BUFFALO program (see Section 2). Photometric redshifts will be derived from such a large photometric baseline by means of state-ofthe-art software such as EAZY (Brammer et al. 2008) and LePhare (Arnouts et al. 1999; Ilbert et al. 2006), fitting galaxy

\footnotetext{
85 http://archive.stsci.edu/

86 http://archive.eso.org/cms/eso-archive-news/first-data-release-from-thegalaxy-clusters-at-vircam-gcav-eso-vista-public-survey.html
}

(and stellar) templates to the spectral energy distribution (SED) of each object. Galaxy physical properties (stellar mass, SFR, etc.) will be inferred by means of an additional SED fitting phase based on stellar population synthesis models (Conroy 2013). Recent studies have found the inclusion of nebular emission to be crucial to estimating physical parameters of galaxies from SED fitting, in particular at high redshift (see, e.g., Schaerer \& de Barros 2012). An additional method combining stellar population models (Bruzual \& Charlot 2003) with nebular emission line models (Gutkin et al. 2016) in an SED fit with the Bayesian code BEAGLE (Chevallard \& Charlot 2016), which is optimized to yield both photometric redshifts and galaxy physical parameters simultaneously, will therefore also be applied to the catalogs. Alternate "datadriven" methods not relying on synthetic templates (see van der Maaten \& Hinton 2008; Van Der Maaten 2014; C. Steinhardt et al. 2020, in preparation) will also be applied to show the potential of novel machine-learning methods in this field of research. The BUFFALO team includes authors of reference SED fitting studies and techniques, as well as HFF luminosity and mass functions (Brammer et al. 2008; Coe et al. 2015; Ishigaki et al. 2015, 2018; Connor et al. 2017; Davidzon et al. 2017; Kawamata et al. 2018), and will use this knowledge to produce a comprehensive "consensus catalog" designed to be used for a wide range of analyses. Moreover, structural parameters for foreground galaxies will be provided via morphological analysis through GALFIT (Peng et al. 2010) and GALAPAGOS (Barden et al. 2012). These codes will be applied with a strategy similar to that used in Morishita et al. (2017).

The A370 catalogs will be released in A. Pagul et al. (2020, in preparation) and A. Niemiec et al. (2020, in preparation), including

1. photometry,

2. photometric redshift (plus spectroscopic redshifts when available) and physical properties,

3. cluster membership,

4. ICL map and structural parameters of foreground galaxies, and

5. mass models as described in Section 7.3

We refer the reader to those papers for further details about the catalog-making process. Catalogs for the other clusters are expected to be released approximately 6 months after their observations are completed (Table 4) and will include the same products as the A370 catalog. 
Table 3

Existing Multiwavelength Frontier Fields Coverage

\begin{tabular}{|c|c|c|c|c|}
\hline Field & Observatory & Wavelengths & Depth & Reference \\
\hline \multirow[t]{9}{*}{ A370 } & VLT/HAWK-I & $2.2 \mu \mathrm{m}$ & $\sim 26.18$ & Brammer et al. (2016) \\
\hline & Spitzer IRAC 1, 2 & $3.6,4.5 \mu \mathrm{m}$ & $\sim 25.19,25.09$ & (PI: T. Soifer and P. Capak) \\
\hline & $\begin{array}{l}\text { Spitzer IRAC 3, } 4 \text { (clus- } \\
\text { ter-only) }\end{array}$ & $5.8,8.0 \mu \mathrm{m}$ & $\sim 23.94,23.39$ & \\
\hline & Spitzer MIPS (cluster-only) & $24 \mu \mathrm{m}$ & $\sim 17.88$ & \\
\hline & Chandra (X-ray) & 515 & 88.0 & \\
\hline & XMM-Newton (X-ray) & 0782150101 & 133.0 & \\
\hline & Bolocam & $140 \mathrm{GHz}$ & $11.8 \mathrm{~h}$ & Sayers et al. (2013) \\
\hline & Planck & $100,143,217,353,545,857 \mathrm{GHz}$ & $\ldots$ & Planck Collaboration et al. (2016b) \\
\hline & Subaru/Suprime-Cam & $B_{\mathrm{J}}, R_{\mathrm{C}}, I_{\mathrm{C}}, i^{\prime}, z^{\prime}$ & $3.24 \mathrm{ks}\left(R_{\mathrm{C}}\right)$ & $\begin{array}{l}\text { von der Linden et al. (2014), } \\
\text { Sereno (2015) }\end{array}$ \\
\hline \multirow[t]{7}{*}{ MACS J0717.5+3745 } & Keck/MOSFIRE & $2.2 \mu \mathrm{m}$ & $\sim 25.31$ & Brammer et al. (2016) \\
\hline & Spitzer IRAC 1, 2, 3, 4 & $3.5,4.5 \mu \mathrm{m}$ & $\sim 25.04,25.17$ & (PI: T. Soifer and P. Capak) \\
\hline & Spitzer IRAC 3,4 & $5.8,8.0 \mu \mathrm{m}$ & $\sim 23.94,23.39$ & \\
\hline & Spitzer MIPS & $24 \mu \mathrm{m}$ & $\sim 17.35$ & \\
\hline & Chandra (X-ray) & 4200 & 58.5 & Donahue et al. (2014) \\
\hline & Chandra (X-ray) & $1655,16235,16305$ & $19.9,70.1,94.3$ & Jauzac et al. (2018) \\
\hline & XMM-Newton (X-ray) & $\begin{array}{c}0672420101,0672420201 \\
0672420301\end{array}$ & $61.2,69.3,64.1$ & Jauzac et al. (2018) \\
\hline \multirow[t]{8}{*}{ MACS J0416.1-2403 } & VLT/HAWK-I & $2.2 \mu \mathrm{m}$ & $\sim 26.25$ & Brammer et al. (2016) \\
\hline & Spitzer & $3.5,4.5 \mu \mathrm{m}$ & $\sim 25.31,25.44$ & (PI: T. Soifer and P. Capak) \\
\hline & Chandra (X-ray) & 10446 & 15.8 & Donahue et al. (2014) \\
\hline & Chandra (X-ray) & $16236,16237,16523$ & $39.9,36.6,71.1$ & $\begin{array}{l}\text { Balestra et al. (2016), Bonamigo et al. } \\
\text { (2018) }\end{array}$ \\
\hline & Chandra (X-ray) & 16304,17313 & $97.8,62.8$ & $\begin{array}{c}\text { Balestra et al. (2016), Bonamigo et al. } \\
\text { (2018) }\end{array}$ \\
\hline & Bolocam & $140 \mathrm{GHz}$ & $7.8 \mathrm{~h}$ & Sayers et al. (2013) \\
\hline & Planck & $100,143,217,353,545,857 \mathrm{GHz}$ & $\ldots$ & Planck Collaboration et al. (2016b) \\
\hline & Subaru/Suprime-Cam & $B_{\mathrm{J}}, R_{\mathrm{C}}, z^{\prime}$ & $\ldots$ & Umetsu et al. (2014), Sereno (2015) \\
\hline \multirow[t]{9}{*}{ Abell S1063 } & VLT/HAWK-I & $2.2 \mu \mathrm{m}$ & $\sim 26.31$ & Brammer et al. (2016) \\
\hline & Spitzer IRAC 1, 2 & $3.6,4.5 \mu \mathrm{m}$ & $\sim 25.04,25.04$ & (PI: T. Soifer and P. Capak) \\
\hline & $\begin{array}{l}\text { Spitzer IRAC 3, } 4 \text { (clus- } \\
\text { ter-only) }\end{array}$ & $5.8,8.0 \mu \mathrm{m}$ & $\sim 22.96,22.64$ & \\
\hline & Spitzer MIPS (cluster-only) & $24 \mu \mathrm{m}$ & $\sim 18.33$ & \\
\hline & Chandra (X-ray) & 4966,3595 & $26.7,19.9$ & Donahue et al. (2014) \\
\hline & Chandra (X-ray) & 18611,18818 & $49.5,47.5$ & Bonamigo et al. (2018) \\
\hline & XMM-Newton (X-ray) & 0504630101 & 52.7 & Lovisari et al. (2017) \\
\hline & Planck & $100,143,217,353,545,857 \mathrm{GHz}$ & $\ldots$ & Planck Collaboration et al. (2016b) \\
\hline & ESO/WFI & $U_{877}, B_{842}, V_{843}, R_{844}, I_{879}, z_{846}$ & $\ldots$ & Umetsu et al. (2014), Sereno (2015) \\
\hline \multirow[t]{8}{*}{ A2744 } & VLT/HAWK-I & $2.2 \mu \mathrm{m}$ & $\sim 26.28$ & Brammer et al. (2016) \\
\hline & Spitzer IRAC 1, 2 & $3.6,4.5 \mu \mathrm{m}$ & $\sim 25.32,25.08$ & (PI: T. Soifer and P. Capak) \\
\hline & $\begin{array}{l}\text { Spitzer IRAC 3, } 4 \text { (clus- } \\
\text { ter-only) }\end{array}$ & $5.8,8.0 \mu \mathrm{m}$ & $\sim 22.78,22.45$ & \\
\hline & Spitzer MIPS (cluster-only) & $24 \mu \mathrm{m}$ & $\sim 18.23$ & \\
\hline & XMM-Newton (X-ray) & 0743850101 & 111.9 & Eckert et al. (2015) \\
\hline & Bolocam & $140 \mathrm{GHz}$ & $\ldots$ & Sayers et al. (2016) \\
\hline & Planck & $100,143,217,353,545,857 \mathrm{GHz}$ & $\ldots$ & Planck Collaboration et al. (2016b) \\
\hline & Subaru/Suprime-Cam & $B_{\mathrm{J}}, R_{\mathrm{C}}, i^{\prime}, z^{\prime}$ & $3.12 \mathrm{ks}\left(R_{\mathrm{C}}\right)$ & Sereno (2015), Medezinski et al. (2016) \\
\hline \multirow[t]{9}{*}{ MACS J1149.5+2223 } & Keck/MOSFIRE & $2.2 \mu \mathrm{m}$ & $\sim 25.41$ & Brammer et al. (2016) \\
\hline & Spitzer & $3.5,4.5 \mu \mathrm{m}$ & $\sim 25.24,25.01$ & (PI: T. Soifer and P. Capak) \\
\hline & Chandra (X-ray) & 3589 & 20.0 & Donahue et al. (2014) \\
\hline & Chandra (X-ray) & $1656,16238,16239,16306$ & $\begin{array}{l}18.5,35.6 \\
51.4,79.7\end{array}$ & \\
\hline & Chandra (X-ray) & $16582,17595,17596$ & $18.8,69.2,72.1$ & \\
\hline & XMM-Newton (X-ray) & 0693661701 & 28.9 & Lovisari et al. (2017) \\
\hline & Bolocam & $140 \mathrm{GHz}$ & $17.7 \mathrm{~h}$ & Sayers et al. (2013) \\
\hline & Planck & $100,143,217,353,545,857 \mathrm{GHz}$ & $\ldots$ & Planck Collaboration et al. (2016b) \\
\hline & Subaru/Suprime-Cam & $B_{\mathrm{J}}, V_{\mathrm{J}}, R_{\mathrm{C}}, I_{\mathrm{C}}, i^{\prime}, z^{\prime}$ & $1.94 \mathrm{ks}\left(I_{\mathrm{C}}\right)$ & $\begin{array}{c}\text { Umetsu et al. (2014); } \\
\text { von der Linden et al. (2014), } \\
\text { Sereno (2015) }\end{array}$ \\
\hline
\end{tabular}


Table 4

BUFFALO HST WFC3 and ACS Observing Schedule

\begin{tabular}{|c|c|c|c|c|c|}
\hline Field & Visit Numbers & Main Cluster & Parallel Field & Epoch 1 & Epoch 2 \\
\hline \multirow[t]{2}{*}{ A370 } & $6 \mathrm{~A}-6 \mathrm{H}$ & WFC3 & ACS & 2018 Jul 21-22 & 2018 Aug 21 \\
\hline & $6 \mathrm{I}-6 \mathrm{P}$ & ACS & WFC3 & 2018 Dec 19 & 2019 Jan 7 \\
\hline MACS J0717.5+3745 & $3 \mathrm{I}-3 \mathrm{P}$ & ACS & WFC3 & 2018 Oct $2-3$ & 2018 Nov 22 \\
\hline \multirow[t]{2}{*}{ MACS J0416.1-2403 } & $2 \mathrm{I}-2 \mathrm{P}$ & ACS & WFC3 & $2019 \operatorname{Jan} 7$ & 2019 Feb 7 \\
\hline & $2 \mathrm{~A}-2 \mathrm{H}$ & WFC3 & ACS & 2019 Aug 3 & 2019 Sep 6 \\
\hline Abell S1063 & $5 \mathrm{~A}-5 \mathrm{H}$ & WFC3 & ACS & 2019 Apr 20 & 2019 May 29 \\
\hline & $1 \mathrm{~A}-1 \mathrm{H}$ & WFC3 & ACS & 2019 Oct-Nov & 2019 Nov-Dec \\
\hline \multirow[t]{2}{*}{ MACS J1149.5+2223 } & $4 \mathrm{~A}-4 \mathrm{H}$ & WFC3 & ACS & 2019 Dec & 2020 Jan \\
\hline & $4 \mathrm{I}-4 \mathrm{P}$ & ACS & WFC3 & 2019 Apr & 2020 May \\
\hline
\end{tabular}

\subsection{Mass Models}

The BUFFALO team includes groups that have been responsible for producing a variety of independent mass models for the HFF clusters from earlier data sets (Jauzac et al. 2014, 2015a, 2015b, 2016b, 2018; Johnson et al. 2014; Lam et al. 2014; Richard et al. 2014; Diego et al. 2015a, 2015b, 2016a, 2016b, 2018; Oguri 2015; Sharon \& Johnson 2015; Wang et al. 2015; Williams et al. 2015, 2018; Harvey et al. 2016; Johnson \& Sharon 2016; Kawamata et al. 2016; Sebesta et al. 2016, 2019; Treu et al. 2016; Priewe et al. 2017; Finney et al. 2018; Mahler et al. 2018; Strait et al. 2018; Williams \& Liesenborgs 2019). Each of these groups will generate an independent mass model (e.g., A. Niemiec et al. 2020, in preparation for A370) based upon a variety of fitting methods (see Section 3). In order to analyze the intrinsic properties of background lensed galaxies, the BUFFALO collaboration will follow the HFF philosophy and release highlevel data products through MAST for the scientific community. An effort is being made to release all of the models for each specific cluster simultaneously in order to encourage a comparison between them.

For strong, weak, or joint strong and weak lensing modelings, each independent team from the BUFFALO collaboration will deliver mass, magnification at several redshifts, deflection, convergence, and shear maps, together with their associated error maps, as well as weak lensing catalogs. Finally, an online magnification calculator will also be available for fast magnification and error estimates.

\subsection{Planned Releases}

Data products for each cluster will be released individually, in order to provide the initial data as rapidly as possible. The first release will contain a mosaic, followed later by the valueadded catalogs and mass models. If HST observations follow the current schedule (Section 2), this will result in approximately the release schedule shown in Table 5.

\section{Summary}

The BUFFALO survey will expand the area of HST coverage by approximately a factor of 4 around the six HFFs, a region that already has multiwavelength coverage, including ultradeep Spitzer imaging. BUFFALO covers this region in five filters, WFC3/IR F105W, F125W, and F160W and ACS/ WFC F606W and F814W, with depths chosen based upon
Table 5

BUFFALO Planned Data Releases

\begin{tabular}{|c|c|c|c|}
\hline Field & $\begin{array}{l}\text { Observations } \\
\text { Complete }\end{array}$ & Mosaic & $\begin{array}{l}\text { Catalogs and } \\
\text { Mass Models }\end{array}$ \\
\hline A 370 & 2019 Jan & 2019 Dec & 2020 Jan \\
\hline $\begin{array}{l}\text { MACS } \\
\qquad 0717.5+3745\end{array}$ & 2019 Apr & 2019 Dec & $2020 \mathrm{Apr}$ \\
\hline $\begin{array}{l}\text { MACS } \\
\text { J0416.1-2403 }\end{array}$ & 2019 Sep & $2020 \mathrm{Mar}$ & 2020 Sep \\
\hline Abell S1063 & 2019 Nov & 2020 May & $2020 \mathrm{Nov}$ \\
\hline A2744 & 2019 Dec & 2020 Jun & 2020 Dec \\
\hline $\begin{array}{l}\text { MACS } \\
\qquad \mathrm{J} 1149.5+2223\end{array}$ & 2020 May & $2020 \mathrm{Nov}$ & 2021 May \\
\hline
\end{tabular}

what has been learned from existing surveys in both HFF and other ultradeep fields. As with the original HFF program, this expanded coverage will simultaneously provide new insights into a wide range of problems at both high and low redshift.

The expanded coverage will not only discover many new sources at $z>7$, with the highest-redshift BUFFALO source most likely to lie at $z \sim 9-10$, but will also provide a significant improvement in measurements of cosmic variance. Both will be important for designing observational programs with JWST. The former is necessary because the NIRSPEC field of view is larger than previous HFF coverage but fits within BUFFALO. The latter will be important both for designing JWST surveys and as a test of theoretical models of early galaxy assembly.

The same observations will allow an improvement in the mass models of these clusters, both in their central regions and in cluster outskirts. BUFFALO is to be the first large HST program with an emphasis on studying the dynamics of infalling cluster substructures. Filamentary structures may contain even a majority of the mass and provide critical insights into the dynamics of galaxy assembly and the cosmic web, and these studies will likely be a significant part of the legacy value of BUFFALO. At the same time, the improvement in mass models of the clusters themselves will improve not only our understanding of structure evolution and dark matter physics but also magnification maps and therefore our existing measurements of the highest-redshift galaxy population accessible prior to JWST. 
Finally, BUFFALO holds the possibility of serendipitous discoveries. The most probable would be the discovery of a lensed SN or other lensed high-redshift transient. If one is found, it will allow a major improvement in mass models and related studies for the cluster in which it is found. Because of increased cosmic variance toward high redshift and high mass, it is also possible that BUFFALO will discover a very early galaxy or assembling galaxy protocluster.

The BUFFALO survey is currently scheduled to have observations completed in 2020 May. Data will be released individually as cluster analysis is completed, with the first data from A370 available on MAST concurrent with the publication of this paper. Mosaics will be available on MAST first, with value-added catalogs and a variety of mass and lensing models made available later.

C.S. acknowledges support from the ERC Consolidator Grant funding scheme (project ConTExT, grant No. 648179). The Cosmic Dawn Center is funded by the Danish National Research Foundation under grant No. 140. M.J. is supported by the United Kingdom Research and Innovation (UKRI) Future Leaders Fellowship "Using Cosmic Beasts to uncover the Nature of Dark Matter" (grant No. MR/S017216/1). This project was also supported by the Science and Technology Facilities Council (grant No. ST/L00075X/1). J.A. is supported by the Postgraduate Research Scholarship in Astroparticle Physics/Cosmology at the University of Sydney. A.A. acknowledges the support of the Royal Society through the Wolfson Fellowship program. Y.M.B. acknowledges funding from the EU Horizon 2020 research and innovation program under Marie Skłodowska-Curie grant agreement 747645 (ClusterGal) and the Netherlands Organisation for Scientific Research (NWO) through VENI grant 016.183.011. R.J.B. gratefully acknowledges the support of TOP grant TOP1.16.057. R.C. is supported by the MERAC foundation postdoctoral grant awarded to C.L. and by the Consejo Nacional de Ciencia y Tecnología CONACYT CVU 520137 Scholar 290609 Overseas Scholarship 438594. The work of T.C. was carried out at the Jet Propulsion Laboratory, California Institute of Technology, under a contract with NASA. B.D. acknowledges financial support from NASA through the Astrophysics Data Analysis Program (ADAP), grant No. NNX12AE20G, and the National Science Foundation, grant No. 1716907. J.M.D. acknowledges the support of project PGC2018101814-B-100 (MCIU/AEI/MINECO/FEDER, UE), Ministerio de Ciencia, Investigación y Universidades. A.C.E. acknowledges support from STFC grant ST/P00541/1. S.E. acknowledges a financial contribution from the contracts ASI 2015-046-R.0 and ASI-INAF n.2017-14-H.0 and INAF "Call per interventi aggiuntivi a sostegno della ricerca di main stream di INAF." O.G. is supported by an NSF Astronomy and Astrophysics Fellowship under award AST-1602595. D.H. acknowledges the support by the Delta ITP foundation. E.J. acknowledges AMU, CNRS, and CNES for their support. C.D.P.L. is funded by the ARC Centre of Excellence for All Sky Astrophysics in 3 Dimensions (ASTRO 3D) through project number CE170100013. M.L. acknowledges CNRS and CNES for support. G.E.M. acknowledges support from the Villum Fonden research grant 13160 "Gas to stars, stars to dust: tracing star formation across cosmic time." The work of L.A.M. took place at the Jet Propulsion Laboratory, California Institute of Technology, under a contract with NASA. J.R. was supported by JPL, which is run under a contract for NASA by Caltech. R.M.R. acknowledges support from HST-GO-15117.005. M.S. is supported by the Netherlands Organization for Scientific Research (NWO) VENI grant 639.041.749 H.Y.S. acknowledges support from the NSFC of China under grant 11973070 and the Shanghai Committee of Science and Technology grant No. 19ZR1466600. G.P.S. acknowledges financial support from STFC grant No. ST/ N000633/1. C.T. acknowledges the support of the Deutsche Forschungsgemeinschaft under BA 1369/28-1 S.T. acknowledges support from the ERC Consolidator Grant funding scheme (project ConTExT, grant No. 648179). The Cosmic Dawn Center is funded by the Danish National Research Foundation under grant No. 140. K.U. was supported by the Ministry of Science and Technology of Taiwan (grant MOST 106-2628-M-001-003-MY3) and the Academia Sinica Investigator Award (grant AS-IA-107M01). R.J.v.W. acknowledges support from the VIDI research program with project No. 639.042.729, which is financed by the Netherlands Organisation for Scientific Research (NWO). This work has made use of the CANDIDE Cluster at the Institut d'Astrophysique de Paris and made possible by grants from the PNCG and the DIM-ACAV.

\section{ORCID iDs}

Charles L. Steinhardt (ib https://orcid.org/0000-00033780-6801

Mathilde Jauzac (ib https://orcid.org/0000-0003-1974-8732 Ana Acebron (iD https://orcid.org/0000-0003-3108-9039

Hakim Atek (1D https://orcid.org/0000-0002-7570-0824 Peter Capak (1D https://orcid.org/0000-0003-3578-6843 Iary Davidzon (iD https://orcid.org/0000-0002-2951-7519 Dominique Eckert (1) https://orcid.org/0000-0001-7917-3892 David Harvey (1D https://orcid.org/0000-0002-6066-6707 Anton M. Koekemoer (i) https://orcid.org/0000-00026610-2048

Claudia D. P. Lagos (ib https://orcid.org/0000-00033021-8564

Guillaume Mahler (iD https://orcid.org/0000-0003-3266-2001

Mireia Montes (ib https://orcid.org/0000-0001-7847-0393 Mario Nonino (iD https://orcid.org/0000-0001-6342-9662 P. A. Oesch (10 https://orcid.org/0000-0001-5851-6649 Johan Richard (iD https://orcid.org/0000-0001-5492-1049 Steven A. Rodney (iD https://orcid.org/0000-0003-1947-687X Matthieu Schaller (D) https://orcid.org/0000-0002-2395-4902 Keren Sharon (ib https://orcid.org/0000-0002-7559-0864 Louis-Gregory Strolger (iD https://orcid.org/0000-00027756-4440

Joseph Allingham (iD https://orcid.org/0000-0003-2718-8640 Adam Amara (iD https://orcid.org/0000-0003-3481-3491 Yannick Bahé (iD https://orcid.org/0000-0002-3196-5126 Céline Bœhm (iD https://orcid.org/0000-0002-5074-9998 Sownak Bose (iD https://orcid.org/0000-0002-0974-5266 Rychard J. Bouwens (D) https://orcid.org/0000-00024989-2471

Larry D. Bradley (iD https://orcid.org/0000-0002-7908-9284 Gabriel Brammer (10) https://orcid.org/0000-0003-2680-005X Rodrigo Cañas (iD https://orcid.org/0000-0003-0776-4102 Renyue Cen (iD https://orcid.org/0000-0001-8531-9536 Dan Coe (iD https://orcid.org/0000-0001-7410-7669 Thomas Connor (D) https://orcid.org/0000-0002-7898-7664 Behnam Darvish (iD https://orcid.org/0000-0003-4919-9017 Jose M. Diego (1) https://orcid.org/0000-0001-9065-3926 Harald Ebeling (iD https://orcid.org/0000-0001-8249-2739 
A. C. Edge (1) https://orcid.org/0000-0002-3398-6916

Eiichi Egami iㅏ https://orcid.org/0000-0003-1344-9475

Stefano Ettori (1) https://orcid.org/0000-0003-4117-8617

Andreas L. Faisst (1) https://orcid.org/0000-0002-9382-9832

Brenda Frye (1) https://orcid.org/0000-0003-1625-8009

Lukas J. Furtak (1) https://orcid.org/0000-0001-6278-032X

C. Gómez-Guijarro (1) https://orcid.org/0000-0002-4085-9165

J. D. Remolina González (i) https://orcid.org/0000-00027868-9827

Anthony Gonzalez (10) https://orcid.org/0000-0002-0933-8601

Or Graur (1) https://orcid.org/0000-0002-4391-6137

Daniel Gruen (1) https://orcid.org/0000-0003-3270-7644

Saurabh W. Jha 1 (1) https://orcid.org/0000-0001-8738-6011

Eric Jullo (1) https://orcid.org/0000-0002-9253-053X

Jean-Paul Kneib (i) https://orcid.org/0000-0002-4616-4989

Vasily Kokorev 느 https://orcid.org/0000-0002-5588-9156

David J. Lagattuta (1) https://orcid.org/0000-0002-7633-2883

Anja von der Linden (i) https://orcid.org/0000-0002-3881-7724

Nora B. Linzer (ㅇ) https://orcid.org/0000-0001-8840-2538

Georgios E. Magdis (ㄴ) https://orcid.org/0000-0002-4872-2294

Richard Massey (ib https://orcid.org/0000-0002-6085-3780

Daniel C. Masters (i) https://orcid.org/0000-0001-5382-6138

Curtis McCully (1) https://orcid.org/0000-0001-5807-7893

Sean L. McGee (1) https://orcid.org/0000-0003-3255-3139

Massimo Meneghetti (i) https://orcid.org/0000-0003-

1225-7084

Leonidas A. Moustakas (ib https://orcid.org/0000-00033030-2360

Eric J. Murphy (i) https://orcid.org/0000-0001-7089-7325

Priyamvada Natarajan (1) https://orcid.org/0000-0002-

5554-8896

Mark Neyrinck (i) https://orcid.org/0000-0002-2618-5790

Masamune Oguri (1) https://orcid.org/0000-0003-3484-399X

Amanda Pagul (i) https://orcid.org/0000-0002-6015-8614

Jason Rhodes (i) https://orcid.org/0000-0002-4485-8549

R. Michael Rich (1) https://orcid.org/0000-0003-0427-8387

Andrew Robertson (1) https://orcid.org/0000-0002-0086-0524

Mauro Sereno (1) https://orcid.org/0000-0003-0302-0325

Graham P. Smith (1) https://orcid.org/0000-0003-4494-8277

Céline Tchernin (1) https://orcid.org/0000-0003-4219-3683

Sune Toft (ㄷ) https://orcid.org/0000-0003-3631-7176

Keiichi Umetsu (i) https://orcid.org/0000-0002-7196-4822

John R. Weaver (1) https://orcid.org/0000-0003-1614-196X

R. J. van Weeren (i) https://orcid.org/0000-0002-0587-1660

Liliya L. R. Williams (i) https://orcid.org/0000-0002-6039-8706

Tom J. Wilson (1) https://orcid.org/0000-0001-6352-9735

Lin Yan (1) https://orcid.org/0000-0003-1710-9339

Adi Zitrin 누 https://orcid.org/0000-0002-0350-4488

\section{References}

Acebron, A., Jullo, E., Limousin, M., et al. 2017, MNRAS, 470, 1809 Adelberger, K. L., Steidel, C. C., Giavalisco, M., et al. 1998, ApJ, 505, 18 Aragon Calvo, M. A., Neyrinck, M. C., \& Silk, J. 2019, OJAp, 2, 7 Arnouts, S., Cristiani, S., Moscardini, L., et al. 1999, MNRAS, 310, 540 Ashby, M. L. N., Caputi, K. I., Cowley, W., et al. 2018, ApJS, 237, 39 Ashby, M. L. N., Willner, S. P., Fazio, G. G., et al. 2015, ApJS, 218, 33 Bahé, Y. M., Barnes, D. J., Dalla Vecchia, C., et al. 2017, MNRAS, 470, 4186 Bahé, Y. M., \& McCarthy, I. G. 2015, MNRAS, 447, 969

Bahé, Y. M., McCarthy, I. G., Balogh, M. L., \& Font, A. S. 2013, MNRAS, 430, 3017

Balestra, I., Mercurio, A., Sartoris, B., et al. 2016, ApJS, 224, 33

Balogh, M. L., Navarro, J. F., \& Morris, S. L. 2000, ApJ, 540, 113

Barbary, K., Aldering, G., Amanullah, R., et al. 2012, ApJ, 745, 31
Barden, M., Häußler, B., Peng, C. Y., McIntosh, D. H., \& Guo, Y. 2012, MNRAS, 422, 449

Barnes, D. J., Kay, S. T., Bahé, Y. M., et al. 2017, MNRAS, 471, 1088

Beckwith, S. V. W., Stiavelli, M., Koekemoer, A. M., et al. 2006, AJ, 132, 1729

Behroozi, P., Wechsler, R., Hearin, A., \& Conroy, C. 2019, MNRAS, 488,3143

Behroozi, P. S., \& Silk, J. 2015, ApJ, 799, 32

Bonamigo, M., Grillo, C., Ettori, S., et al. 2018, ApJ, 864, 98

Bond, J. R., Kofman, L., \& Pogosyan, D. 1996, Natur, 380, 603

Bouwens, R. J., Illingworth, G. D., Oesch, P. A., et al. 2015, ApJ, 803, 34

Bouwens, R. J., Oesch, P. A., Labbé, I., et al. 2016, ApJ, 830, 67

Bradač, M., Allen, S. W., Treu, T., et al. 2008, ApJ, 687, 959

Bradač, M., Clowe, D., Gonzalez, A. H., et al. 2006, ApJ, 652, 937

Bradač, M., Ryan, R., Casertano, S., et al. 2014, ApJ, 785, 108

Brammer, G. B., Marchesini, D., Labbé, I., et al. 2016, ApJS, 226, 6

Brammer, G. B., van Dokkum, P. G., \& Coppi, P. 2008, ApJ, 686, 1503

Bridge, J. S., Holwerda, B. W., Stefanon, M., et al. 2019, ApJ, 882, 42

Broadhurst, T., Takada, M., Umetsu, K., et al. 2005, ApJL, 619, L143

Bruzual, G., \& Charlot, S. 2003, MNRAS, 344, 1000

Canas, R., Elahi, P. J., Welker, C., et al. 2019a, MNRAS, 482, 2039

Canas, R., Elahi, P. J., Welker, C., et al. 2019b, arXiv:1908.02945

Cen, R., Roxana Pop, A., \& Bahcall, N. A. 2014, PNAS, 111, 7914

Chen, W., Kelly, P. L., Diego, J. M., et al. 2019, ApJ, 881, 8

Chevallard, J., \& Charlot, S. 2016, MNRAS, 462, 1415

Chiu, I. N., Umetsu, K., Sereno, M., et al. 2018, ApJ, 860, 126

Clowe, D., Bradač, M., Gonzalez, A. H., et al. 2006, ApJL, 648, L109

Clowe, D., Gonzalez, A., \& Markevitch, M. 2004, ApJ, 604, 596

Coe, D., Bradley, L., \& Zitrin, A. 2015, ApJ, 800, 84

Coe, D., Salmon, B., Bradac, M., et al. 2019, ApJ, 884, 85

Connor, T., Donahue, M., Kelson, D. D., et al. 2017, ApJ, 848, 37

Connor, T., Kelson, D. D., Donahue, M., \& Moustakas, J. 2019, ApJ, 875, 16

Connor, T., Kelson, D. D., Mulchaey, J., et al. 2018, ApJ, 867, 25

Conroy, C. 2013, ARA\&A, 51, 393

Conroy, C., Wechsler, R. H., \& Kravtsov, A. V. 2007, ApJ, 668, 826

Contini, E., De Lucia, G., Villalobos, Á., \& Borgani, S. 2014, MNRAS, 437, 3787

Cui, W., Murante, G., Monaco, P., et al. 2014, MNRAS, 437, 816

Daddi, E., Dickinson, M., Morrison, G., et al. 2007, ApJ, 670, 156

Davidzon, I., Ilbert, O., Laigle, C., et al. 2017, A\&A, 605, A70

De Lucia, G., Weinmann, S., Poggianti, B. M., Aragón-Salamanca, A., \& Zaritsky, D. 2012, MNRAS, 423, 1277

DeMaio, T., Gonzalez, A. H., Zabludoff, A., et al. 2018, MNRAS, 474, 3009

DeMaio, T., Gonzalez, A. H., Zabludoff, A., Zaritsky, D., \& Bradač, M. 2015, MNRAS, 448, 1162

Diego, J. M., Broadhurst, T., Chen, C., et al. 2016a, MNRAS, 456, 356

Diego, J. M., Broadhurst, T., Molnar, S. M., Lam, D., \& Lim, J. 2015a, MNRAS, 447, 3130

Diego, J. M., Broadhurst, T., Wong, J., et al. 2016b, MNRAS, 459, 3447

Diego, J. M., Broadhurst, T., Zitrin, A., et al. 2015b, MNRAS, 451, 3920

Diego, J. M., Schmidt, K. B., Broadhurst, T., et al. 2018, MNRAS, 473, 4279

Diego, J. M., Tegmark, M., Protopapas, P., \& Sandvik, H. B. 2007, MNRAS, 375,958

Dietrich, J. P., Werner, N., Clowe, D., et al. 2012, Natur, 487, 202

Donahue, M., Voit, G. M., Mahdavi, A., et al. 2014, ApJ, 794, 136

Dressler, A. 1980, ApJ, 236, 351

Eckert, D., Jauzac, M., Shan, H., et al. 2015, Natur, 528, 105

Ellis, R. S., McLure, R. J., Dunlop, J. S., et al. 2013, ApJL, 763, L7

Fan, Z., Shan, H., \& Liu, J. 2010, ApJ, 719, 1408

Finkelstein, S. L., Song, M., Behroozi, P., et al. 2015, ApJ, 814, 95

Finney, E. Q., Bradač, M., Huang, K.-H., et al. 2018, ApJ, 859, 58

Frye, B. L., Pascale, M., Qin, Y., et al. 2019, ApJ, 871, 51

Giavalisco, M., Ferguson, H. C., Koekemoer, A. M., et al. 2004, ApJL, 600, L93

Goh, T., Primack, J., Lee, C. T., et al. 2019, MNRAS, 483, 2101

Graur, O., Rodney, S. A., Maoz, D., et al. 2014, ApJ, 783, 28

Grillo, C., Rosati, P., Suyu, S. H., et al. 2018, ApJ, 860, 94

Grogin, N. A., Kocevski, D. D., Faber, S. M., et al. 2011, ApJS, 197, 35

Gunn, J. E., Gott, J., \& Richard, I. 1972, ApJ, 176, 1

Gunn, J. E., \& Stryker, L. L. 1983, ApJS, 52, 121

Gutkin, J., Charlot, S., \& Bruzual, G. 2016, MNRAS, 462, 1757

Haines, C. P., Pereira, M. J., Smith, G. P., et al. 2015, ApJ, 806, 101

Harvey, D., Kneib, J. P., \& Jauzac, M. 2016, MNRAS, 458, 660

Harvey, D., Massey, R., Kitching, T., et al. 2013, MNRAS, 433, 1517

Harvey, D., Massey, R., Kitching, T., Taylor, A., \& Tittley, E. 2015, Sci, 347, 1462 
Harvey, D., Tittley, E., Massey, R., et al. 2014, MNRAS, 441, 404 Hashimoto, Y., Oemler, A., Jr., Lin, H., \& Tucker, D. L. 1998, ApJ, 499, 589 Ilbert, O., Arnouts, S., McCracken, H. J., et al. 2006, A\&A, 457, 841 Illingworth, G. D., Magee, D., Oesch, P. A., et al. 2013, ApJS, 209, 6 Ishigaki, M., Kawamata, R., Ouchi, M., et al. 2015, ApJ, 799, 12 Ishigaki, M., Kawamata, R., Ouchi, M., Oguri, M., \& Shimasaku, K. 2018, ApJ, 854, 73

Jansen, F., Lumb, D., Altieri, B., et al. 2001, A\&A, 365, L1

Jauzac, M., Clément, B., Limousin, M., et al. 2014, MNRAS, 443, 1549

Jauzac, M., Eckert, D., Schaller, M., et al. 2018, MNRAS, 481, 2901

Jauzac, M., Eckert, D., Schwinn, J., et al. 2016a, MNRAS, 463, 3876

Jauzac, M., Jullo, E., Eckert, D., et al. 2015a, MNRAS, 446, 4132

Jauzac, M., Jullo, E., Kneib, J.-P., et al. 2012, MNRAS, 426, 3369

Jauzac, M., Richard, J., Jullo, E., et al. 2015b, MNRAS, 452, 1437

Jauzac, M., Richard, J., Limousin, M., et al. 2016b, MNRAS, 457, 2029

Jiménez-Teja, Y., \& Dupke, R. 2016, ApJ, 820, 49

Johnson, T. L., \& Sharon, K. 2016, ApJ, 832, 82

Johnson, T. L., Sharon, K., Bayliss, M. B., et al. 2014, ApJ, 797, 48

Jullo, E., \& Kneib, J.-P. 2009, MNRAS, 395, 1319

Jullo, E., Kneib, J.-P., Limousin, M., et al. 2007, NJPh, 9, 447

Kahlhoefer, F., Schmidt-Hoberg, K., Frandsen, M. T., \& Sarkar, S. 2014 MNRAS, 437, 2865

Kaurov, A. A., Dai, L., Venumadhav, T., Miralda-Escudé, J., \& Frye, B. 2019, ApJ, 880, 58

Kawamata, R., Ishigaki, M., Shimasaku, K., et al. 2018, ApJ, 855, 4

Kawamata, R., Oguri, M., Ishigaki, M., Shimasaku, K., \& Ouchi, M. 2016, ApJ, 819, 114

Kelly, P. L., Diego, J. M., Rodney, S., et al. 2018, NatAs, 2, 334

Kelly, P. L., Rodney, S. A., Treu, T., et al. 2015, Sci, 347, 1123

Kelly, P. L., Rodney, S. A., Treu, T., et al. 2016, ApJL, 819, L8

Koekemoer, A. M., Ellis, R. S., McLure, R. J., et al. 2013, ApJS, 209, 3

Koekemoer, A. M., Faber, S. M., Ferguson, H. C., et al. 2011, ApJS, 197, 36

Labbé, I., Oesch, P. A., Bouwens, R. J., et al. 2013, ApJL, 777, L19

Labbé, I., Oesch, P. A., Illingworth, G. D., et al. 2015, ApJS, 221, 23

Lagattuta, D. J., Richard, J., Bauer, F. E., et al. 2019, MNRAS, 485, 3738

Lagos, C. d. P., Tobar, R. J., Robotham, A. S. G., et al. 2018, MNRAS, 481, 3573

Laigle, C., Pichon, C., Arnouts, S., et al. 2018, MNRAS, 474, 5437

Lam, D., Broadhurst, T., Diego, J. M., et al. 2014, ApJ, 797, 98

Laporte, N., Streblyanska, A., Clement, B., et al. 2014, A\&A, 562, L8

Lee, N., Sanders, D. B., Casey, C. M., et al. 2013, ApJ, 778, 131

Liesenborgs, J., De Rijcke, S., \& Dejonghe, H. 2006, MNRAS, 367, 1209

Liesenborgs, J., de Rijcke, S., Dejonghe, H., \& Bekaert, P. 2009, MNRAS, 397,341

Limousin, M., Richard, J., Jullo, E., et al. 2007, ApJ, 668, 643

Liu, F. S., Jia, M., Yesuf, H. M., et al. 2018, ApJ, 860, 60

Lotz, J. M., Koekemoer, A., Coe, D., et al. 2017, ApJ, 837, 97

Lovisari, L., Forman, W. R., Jones, C., et al. 2017, ApJ, 846, 51

Mahler, G., Richard, J., Clément, B., et al. 2018, MNRAS, 473, 663

Malavasi, N., Arnouts, S., Vibert, D., et al. 2017, MNRAS, 465, 3817

Marian, V., Ziegler, B., Kuchner, U., \& Verdugo, M. 2018, A\&A, 617, A34

Martinet, N., Durret, F., Adami, C., \& Rudnick, G. 2017, A\&A, 604, A80

Mashian, N., Oesch, P. A., \& Loeb, A. 2016, MNRAS, 455, 2101

Massey, R., Kitching, T., \& Nagai, D. 2011, MNRAS, 413, 1709

McLure, R. J., Dunlop, J. S., Cirasuolo, M., et al. 2010, MNRAS, 403, 960

Medezinski, E., Umetsu, K., Okabe, N., et al. 2016, ApJ, 817, 24

Meneghetti, M., Natarajan, P., Coe, D., et al. 2017, MNRAS, 472, 3177

Merten, J., Coe, D., Dupke, R., et al. 2011, MNRAS, 417, 333

Mihos, J. C. 2004, in Proc. Carnegie Observatories Centennial Symp., Clusters of Galaxies: Probes of Cosmological Structure and Galaxy Evolution, ed. J. S. Mulchaey, A. Dressler, \& A. Oemler (Cambridge: Cambridge Univ. Press), 277

Mohammed, I., Saha, P., Williams, L. L. R., Liesenborgs, J., \& Sebesta, K 2016, MNRAS, 459, 1698

Montes, M. 2019, arXiv:1912.01616

Montes, M., \& Trujillo, I. 2014, ApJ, 794, 137

Montes, M., \& Trujillo, I. 2018, MNRAS, 474, 917

Montes, M., \& Trujillo, I. 2019, MNRAS, 482, 2838

Moore, B., Katz, N., Lake, G., Dressler, A., \& Oemler, A. 1996, Natur, 379,613

Morishita, T., Abramson, L. E., Treu, T., et al. 2017, ApJ, 846, 139

Morishita, T., Trenti, M., Stiavelli, M., et al. 2018, ApJ, 867, 150

Moss, C. 2006, MNRAS, 373, 167

Moster, B. P., Somerville, R. S., Newman, J. A., \& Rix, H.-W. 2011, ApJ, 731,113

Murante, G., Giovalli, M., Gerhard, O., et al. 2007, MNRAS, 377, 2
Natarajan, P., Loeb, A., Kneib, J.-P., \& Smail, I. 2002, ApJL, 580, L17

Neyrinck, M. C., Hidding, J., Konstantatou, M., \& van de Weygaert, R. 2018, RSOS, 5, 171582

Niemiec, A., Jullo, E., Giocoli, C., Limousin, M., \& Jauzac, M. 2019, MNRAS, 487, 653

Noeske, K. G., Weiner, B. J., Faber, S. M., et al. 2007, ApJL, 660, L43

Nordin, J., Rubin, D., Richard, J., et al. 2014, MNRAS, 440, 2742

Oesch, P. A., Bouwens, R. J., Carollo, C. M., et al. 2010, ApJL, 725, L150

Oesch, P. A., Bouwens, R. J., Illingworth, G. D., et al. 2014, ApJ, 786, 108

Oesch, P. A., Brammer, G., van Dokkum, P. G., et al. 2016, ApJ, 819, 129

Oesch, P. A., Labbé, I., Bouwens, R. J., et al. 2013, ApJ, 772, 136

Oguri, M. 2010, PASJ, 62, 1017

Oguri, M. 2015, MNRAS, 449, L86

Oke, J. B. 1974, ApJS, 27, 21

Olave-Rojas, D., Cerulo, P., Demarco, R., et al. 2018, MNRAS, 479, 2328

O’Shea, B. W., Wise, J. H., Xu, H., \& Norman, M. L. 2015, ApJL, 807, L12

Owers, M. S., Couch, W. J., Nulsen, P. E. J., \& Rand all, S. W. 2012, ApJL, 750, L23

Patel, B., McCully, C., Jha, S. W., et al. 2014, ApJ, 786, 9

Peng, C. Y., Ho, L. C., Impey, C. D., \& Rix, H.-W. 2010, AJ, 139, 2097

Peng, Y., Maiolino, R., \& Cochrane, R. 2015, Natur, 521, 192

Perez, J., Tissera, P., Padilla, N., Alonso, M. S., \& Lambas, D. G. 2009, MNRAS, 399, 1157

Planck Collaboration, Adam, R., Ade, P. A. R., et al. 2016a, A\&A, 594, A1

Planck Collaboration, Ade, P. A. R., Aghanim, N., et al. 2016b, A\&A, 594, A27

Planck Collaboration, Aghanim, N., Akrami, Y., et al. 2018, arXiv:1807.06209

Poggianti, B. M., Gullieuszik, M., Tonnesen, S., et al. 2019, MNRAS, 482, 4466

Postman, M., Coe, D., Benítez, N., et al. 2012, ApJS, 199, 25

Priewe, J., Williams, L. L. R., Liesenborgs, J., Coe, D., \& Rodney, S. A. 2017, MNRAS, 465, 1030

Puchwein, E., Springel, V., Sijacki, D., \& Dolag, K. 2010, MNRAS, 406, 936

Purcell, C. W., Bullock, J. S., \& Zentner, A. R. 2007, ApJ, 666, 20

Richard, J., Jauzac, M., Limousin, M., et al. 2014, MNRAS, 444, 268

Richard, J., Smith, G. P., Kneib, J.-P., et al. 2010, MNRAS, 404, 325

Roberts-Borsani, G. W., Bouwens, R. J., Oesch, P. A., et al. 2016, ApJ, 823,143

Robertson, A., Harvey, D., Massey, R., et al. 2019, MNRAS, 488, 3646

Robertson, B. E., Ellis, R. S., Dunlop, J. S., et al. 2014, ApJL, 796, L27

Robertson, B. E., Ellis, R. S., Dunlop, J. S., McLure, R. J., \& Stark, D. P. 2010, Natur, 468, 49

Rodney, S. A., Balestra, I., Bradac, M., et al. 2018, NatAs, 2, 324

Rodney, S. A., Patel, B., Scolnic, D., et al. 2015a, ApJ, 811, 70

Rodney, S. A., Riess, A. G., Scolnic, D. M., et al. 2015b, AJ, 150, 156

Rodney, S. A., Riess, A. G., Strolger, L.-G., et al. 2014, AJ, 148, 13

Rodney, S. A., Strolger, L. G., Kelly, P. L., et al. 2016, ApJ, 820, 50

Rodríguez-Muñoz, L., Rodighiero, G., Mancini, C., et al. 2019, MNRAS, 485,586

Rubin, D., Hayden, B., Huang, X., et al. 2018, ApJ, 866, 65

Rudick, C. S., Mihos, J. C., \& McBride, C. K. 2011, ApJ, 732, 48

Sayers, J., Golwala, S. R., Mantz, A. B., et al. 2016, ApJ, 832, 26

Sayers, J., Mroczkowski, T., Zemcov, M., et al. 2013, ApJ, 778, 52

Schaerer, D., \& de Barros, S. 2012, in IAU Symp. 284, The Spectral Energy

Distribution of Galaxies, ed. R. J. Tuffs \& C. C. Popescu (Cambridge: Cambridge Univ. Press), 20

Schmidt, K. B., Treu, T., Trenti, M., et al. 2014, ApJ, 786, 57

Schwinn, J., Jauzac, M., Baugh, C. M., et al. 2017, MNRAS, 467, 2913

Scoville, N., Abraham, R. G., Aussel, H., et al. 2007, ApJS, 172, 38

Sebesta, K., Williams, L. L. R., Liesenborgs, J., Medezinski, E., \& Okabe, N. 2019, MNRAS, 488, 3251

Sebesta, K., Williams, L. L. R., Mohammed, I., Saha, P., \& Liesenborgs, J. 2016, MNRAS, 461, 2126

Sereno, M. 2015, MNRAS, 450, 3665, (CoMaLit-III)

Sereno, M., Umetsu, K., Ettori, S., et al. 2018, ApJL, 860, L4

Sharon, K., \& Johnson, T. L. 2015, ApJL, 800, L26

Speagle, J. S., Steinhardt, C. L., Capak, P. L., \& Silverman, J. D. 2014, ApJS, 214, 15

Springel, V., White, S. D. M., Jenkins, A., et al. 2005, Natur, 435, 629

Stark, D. P., Schenker, M. A., Ellis, R., et al. 2013, ApJ, 763, 129

Steinhardt, C. L., Capak, P., Masters, D., \& Speagle, J. S. 2016, ApJ, 824, 21

Steinhardt, C. L., Speagle, J. S., Capak, P., et al. 2014, ApJL, 791, L25

Strait, V., Bradač, M., Hoag, A., et al. 2018, ApJ, 868, 129

Strolger, L.-G., Dahlen, T., Rodney, S. A., et al. 2015, ApJ, 813, 93

Tonnesen, S., \& Cen, R. 2012, MNRAS, 425, 2313

Trenti, M., Bradley, L. D., Stiavelli, M., et al. 2011, ApJL, 727, L39 
Trenti, M., Bradley, L. D., Stiavelli, M., et al. 2012, ApJ, 746, 55

Treu, T., Brammer, G., Diego, J. M., et al. 2016, ApJ, 817, 60

Treu, T., Schmidt, K. B., Brammer, G. B., et al. 2015, ApJ, 812, 114

Umetsu, K. 2013, ApJ, 769, 13

Umetsu, K., Medezinski, E., Nonino, M., et al. 2014, ApJ, 795, 163

Umetsu, K., Sereno, M., Tam, S.-I., et al. 2018, ApJ, 860, 104

Umetsu, K., Zitrin, A., Gruen, D., et al. 2016, ApJ, 821, 116

Van Der Maaten, L. 2014, J. Mach. Learn. Res., 15, 3221

van der Maaten, L., \& Hinton, G. 2008, J. Mach. Learn. Res., 9, 2579

Villalobos, Á., De Lucia, G., Borgani, S., \& Murante, G. 2012, MNRAS, 424, 2401

Vogelsberger, M., Genel, S., Springel, V., et al. 2014, Natur, 509, 177

von der Linden, A., Allen, M. T., Applegate, D. E., et al. 2014, MNRAS, 439,2

Vulcani, B., Treu, T., Schmidt, K. B., et al. 2016, ApJ, 833, 178
Wagner, C. R., Courteau, S., Brodwin, M., et al. 2017, ApJ, 834, 53

Wang, X., Hoag, A., Huang, K. H., et al. 2015, ApJ, 811, 29

Weisskopf, M. C., Tananbaum, H. D., Van Speybroeck, L. P., \& O’Dell, S. L. 2000, Proc. SPIE, 4012, 2

Werner, M. W., Roellig, T. L., Low, F. J., et al. 2004, ApJS, 154, 1

Williams, L. L. R., \& Liesenborgs, J. 2019, MNRAS, 482, 5666

Williams, L. L. R., Sebesta, K., \& Liesenborgs, J. 2018, MNRAS, 480, 3140

Williams, L. L. R., Sebesta, K., Saha, P., Mohammed, I., \& Liesenborgs, J. 2015, IAUGA, 29, 2255413

Wittman, D., Golovich, N., \& Dawson, W. A. 2018, ApJ, 869, 104

Yan, H., Yan, L., Zamojski, M. A., et al. 2011, ApJL, 728, L22

Zheng, W., Shu, X., Moustakas, J., et al. 2014, ApJ, 795, 93

Zitrin, A., Broadhurst, T., Umetsu, K., et al. 2009, MNRAS, 396, 1985

Zitrin, A., Fabris, A., Merten, J., et al. 2015, ApJ, 801, 44 